30 Iman Osman, MD

31 NYU School of Medicine

$32 \quad 522$ First Avenue

33 Phone: 212-263-9075; Fax: 212-263-9090

34 Aristotelis Tsirigos ${ }^{2,3,4 *}$

${ }^{2}$ Interdisciplinary Melanoma Cooperative Group

${ }^{3}$ Applied Bioinformatics Laboratories

${ }^{4}$ Department of Pathology

${ }^{5}$ Department of Medicine

${ }^{6}$ Deparmtent of Radiology

${ }^{7}$ Department of Population Health

${ }^{8}$ Department of Surgery

${ }^{9}$ Skirball Institute Department of Cell Biology

$\dagger$ These authors contributed equally to this work.

${ }^{*}$ Corresponding Authors:

Aristotelis Tsirigos, $\mathrm{PhD}$

Associate Professor of Pathology

Director, Applied Bioinformatics Laboratories

New York University School of Medicine

227 East $30^{\text {th }}$ Street

New York, New York

Phone: 646-501-2693

Email: Aristotelis.Tsirigos@nyulangone.org

Email: Iman.Osman@nyulangone.org

\title{
A Deep Learning Approach for Rapid Mutational Screening in Melanoma
}

Randie H. Kim ${ }^{1,2 \dagger}$, Sofia Nomikou ${ }^{3 \dagger}$, Zarmeena Dawood ${ }^{2}$, George Jour ${ }^{1,2,4}$, Douglas Donnelly ${ }^{2}$, Una Moran², Jeffrey S. Weber ${ }^{2,5}$, Narges Razavian ${ }^{6,7}$, Matija Snuderl ${ }^{4}$, Richard Shapiro ${ }^{2,8}$, Russell S. Berman ${ }^{2,8}$, Nicolas Coudray ${ }^{3,9}$, Iman Osman ${ }^{1,2,5 *}$,

${ }^{1}$ The Ronald O. Perelman Department of Dermatology

New York University School of Medicine, New York, New York. 


\section{Abstract}

37 DNA-based molecular assays for determining mutational status in melanomas are timeconsuming and costly. As an alternative, we applied a deep convolutional neural network

39 (CNN) to histopathology images of tumors from 257 melanoma patients and developed a

40 fully automated model that first selects for tumor-rich areas (Area under the curve $A \cup C=0.98)$, and second, predicts for the presence of mutated BRAF or NRAS. Network

42 performance was enhanced on $B R A F$-mutated melanomas $\leq 1.0 \mathrm{~mm}(\mathrm{AUC}=0.83)$ and on

43 non-ulcerated NRAS-mutated melanomas (AUC=0.92). Applying our models to

44 histological images of primary melanomas from The Cancer Genome Atlas database also

45 demonstrated improved performances on thinner BRAF-mutated melanomas and non-

46 ulcerated NRAS-mutated melanomas. We propose that deep learning-based analysis of

47 histological images has the potential to become integrated into clinical decision making

48 for the rapid detection of mutations of interest in melanoma. 


\section{Introduction}

Mutations in the BRAF oncogene are found in $50-60 \%$ of all melanomas ${ }^{1}$, while NRAS mutations comprise an additional $15-20 \%$. With the development of targeted therapies ${ }^{2}$, ${ }^{3}$, determining the mutational status of BRAF and NRAS has become an integral component for the management of Stage III/IV melanomas. DNA molecular assays such as Sanger sequencing, pyrosequencing, and next generation sequencing (NGS) are the current gold standard to determine mutational status ${ }^{4}$. However, these methods are costly and time-consuming. Immunohistochemistry, real-time polymerase chain reaction (PCR), and automated platforms $s^{5,6}$ are rapid and less expensive alternatives, but are limited to screening for specific mutations, such as BRAF-V600E/K or NRAS-Q61R/L, and may potentially fail to identify rare mutational variants in patients that might have otherwise benefited from adjuvant targeted therapy.

Deep Convolutional Neural Network (CNN) methods to predict mutational status have been demonstrated in other solid tumors. CNNs utilize multiple layers of convolution operations, pooling layers, and fully connected layers to perform classification of images to classes of interest through identification of various image features often not directly detectable by the human eye. Deep CNNs, which utilize non-linear learning algorithms, have been successful in manipulating and processing large data sets, particularly for image analysis ${ }^{8}$. Using images from The Cancer Genome Atlas (TCGA), a collaborative cancer genomics database ${ }^{9}$, our group has previously developed a machine learning algorithm that can predict for 6 different genes, including EGFR and STK11, in lung carcinoma $^{10}$. In breast cancer, deep learning applied to tumor microarray images has been shown to predict for $E R$ status with an $84 \%$ accuracy ${ }^{11}$. 
75 In this study, we adapt our previous deep learning algorithm to a different dataset comprised of histopathology images of primary melanomas resected from patients prospectively enrolled in a single-institution IRB-approved clinicopathological and biorepository in order to develop a model from tissue specimens that are more representative of what might be seen in routine clinical practice. While molecular testing is typically performed on the most recent metastatic sample, testing on the primary tumor can be performed if metastatic tissue is unavailable or carries a low tumor burden ${ }^{12,13}$. We present our deep learning models for the screening of BRAF and NRAS mutations in primary melanomas, with the purpose of exploring its potential clinical utility.

\section{Results}

\section{Patient characteristics}

324 primary melanomas from 266 unique patients were included in this study and divided into training $(n=182)$, validation $(n=43)$, and independent $(n=41)$ cohorts, without overlap between the patient subsets. Within each cohort, BRAF-mutant, NRAS-mutant, and WT/WT melanomas were represented (Table 1). The average ages of patients with BRAF-mutant melanoma were 59.4, 53.9, and 59-years old in training, validation, and independent cohorts, respectively. For patients with NRAS-mutant melanoma, the average ages were $61.2,70.9$, and 59.9-years old; and for WT/WT patients, the average ages were 65.1, 68.5, and 65.1-years old. 


\section{Dataset characteristics}

365 formalin-fixed paraffin embedded (FFPE) hematoxylin and eosin (H\&E)-stained slides from 324 primary melanomas were digitized and reviewed for quality control. After excluding images that were blurry, faded, or contained no tumor, 293 images from 257 melanomas were available for analysis. 103 BRAF-mutant, 94 NRAS-mutant, and 96 WT/WT melanomas images were included in the study. V600E comprised $70 \%$ of the BRAF mutations. NRAS Q61R/Q61K comprised $80 \%$ of the NRAS mutations.

\section{Computational workflow for whole-slide histopathology image analysis}

Our computational workflow with the CNN Inception v3 is shown in Fig. 1. and is common across all our classifiers (see Methods). The aim of our analytical approach was to: (1) to predict the presence of BRAF and NRAS mutations using manually annotated slides; and (2) to automate the annotation process by the CNN.

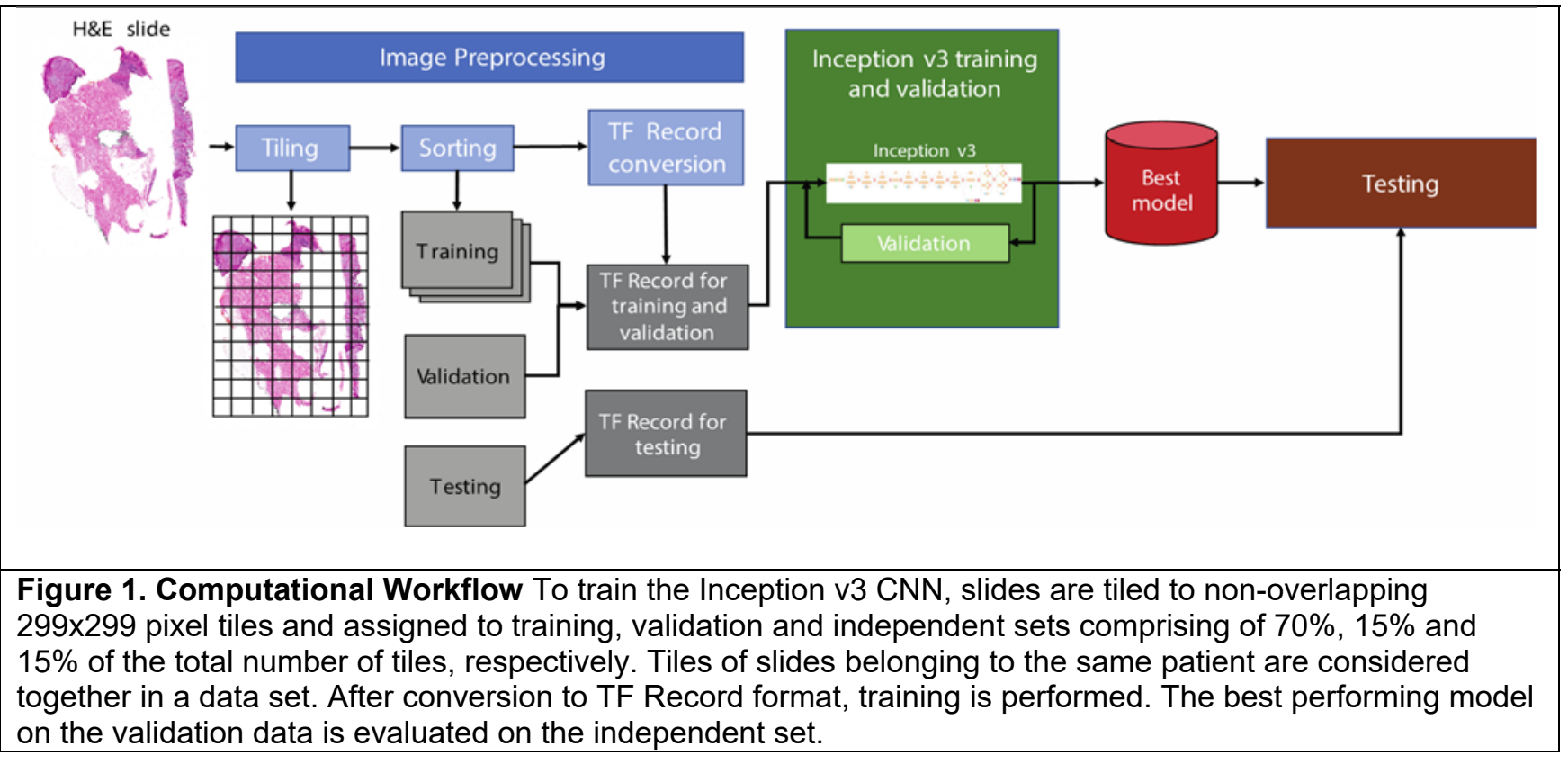

Figure 1. Computational Workflow To train the Inception v3 CNN, slides are tiled to non-overlapping $299 \times 299$ pixel tiles and assigned to training, validation and independent sets comprising of $70 \%, 15 \%$ and $15 \%$ of the total number of tiles, respectively. Tiles of slides belonging to the same patient are considered on the validation data is evaluated on the independent set. 
112 Predicting BRAF and NRAS mutation on manually annotated whole-slide images

113 Because of tissue heterogeneity in skin specimens, tumor-rich areas were initially

114 manually annotated as regions of interest (ROI). Normal skin and associated

115 appendages, connective and subcutaneous tissue, necrosis, hemorrhage, and

116 aggregates of dense inflammation were excluded from training. The network was trained

117 on tiled images of manually annotated ROI, with $70 \%$ of images used for training, $15 \%$

118 used for validation, and $15 \%$ used for independent testing (Supplemental Table 1).

119 Model performance achieved a per slide Area Under the Curve (AUC) $=0.75[95 \% \mathrm{Cl}$ :

$120 \quad 0.60,0.90]$ for predicting $B R A F^{\text {Mut }}$ (Supplemental Figure 1) and $A U C=0.77[95 \% \mathrm{Cl}$ :

$121 \quad 0.58,0.96]$ for predicting NRAS ${ }^{M u t}$ (Supplemental Figure 2).

122

123 We next sought to elucidate some of the parameters that could influence network

124 performance. To evaluate the role of tumor thickness, tumors from the independent cohort

125 were sorted by Breslow depth. Model performance for predicting mutated BRAF improved

126 for slides with a tumor thickness $\leq 1.0 \mathrm{~mm}$, with an $\mathrm{AUC}=0.83$ [95\% Cl: 0.45,1.0] (Fig. 2A,

127 left). Conversely, there were reductions in the AUC to 0.74 [95\% Cl: $0.58,0.89]$ for tumors

$128>1.0-5.0 \mathrm{~mm}$, and to $0.75[95 \% \mathrm{Cl}: 0.60,0.90]$ for very thick tumors $>5.0-10 \mathrm{~mm}$. One

129 potential explanation for this difference is that BRAF-mutated melanomas are associated

130 with a distinctive epidermal component, such as increased pagetoid scatter and

131 intraepidermal nesting of melanocytes ${ }^{14}$. These histologic features may carry more weight

132 in thinner tumors compared to deeper and more invasive melanomas. NRAS mutation

133 prediction was not consistently dependent on tumor thickness (Fig 2B, left;

134 Supplemental Table 2). 
A

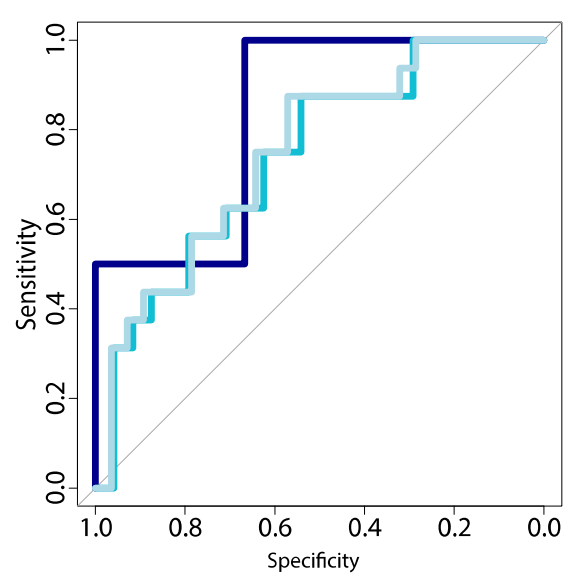

$-<=1 \mathrm{~mm}, \mathrm{AUC}=0.83$

$<=5 \mathrm{~mm}, \mathrm{AUC}=0.74$

B

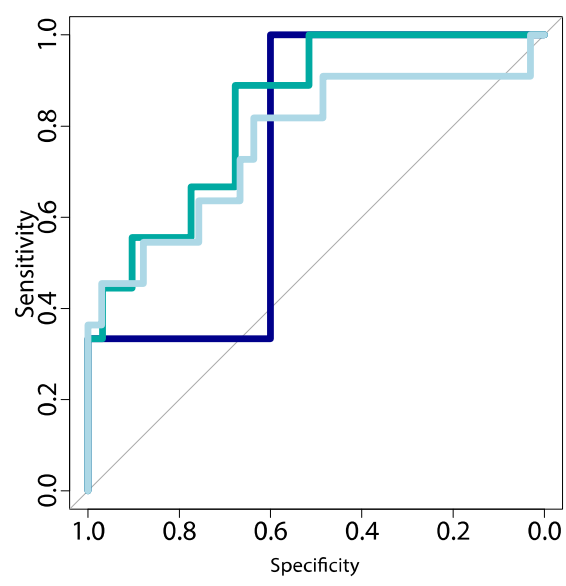

$-<=1 \mathrm{~mm}, \mathrm{AUC}=0.73$
$-<=5 \mathrm{~mm}, \mathrm{AUC}=0.84$
$<=10 \mathrm{~mm}, \mathrm{AUC}=0.77$
Ulceration

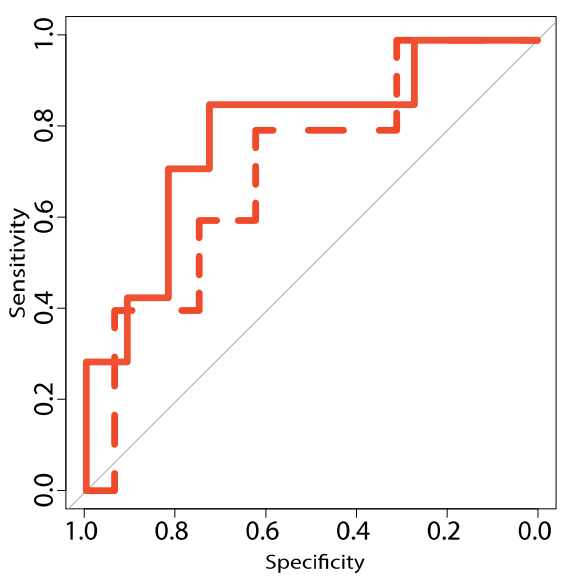

- Absent, AUC $=0.71$

Present, $A \cup C=0.79$

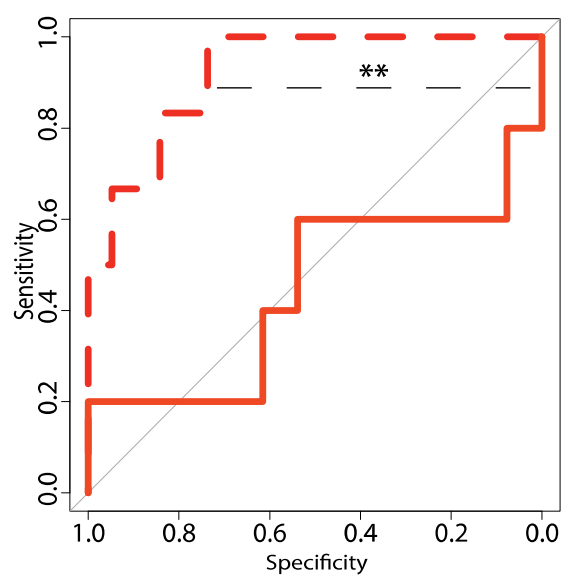

- Absent, AUC $=0.92$

Present, AUC $=0.45$

Figure 2. Exploration of parameters contributing to network performance A) BRAF mutation prediction. ROC plots for melanomas $\leq 1.0 \mathrm{~mm},>1.0-5.0 \mathrm{~mm}$ and $>5.0-10 \mathrm{~mm}$ with the network trained on manually annotated slides demonstrate improved performance for thinner tumors (left). ROC plots for melanomas based on ulceration status show improved performance if ulceration was present (right). B) NRAS mutation prediction. ROC curves for melanomas based on Breslow depth show no significant differences (left). NRAS mutation prediction is improved for melanomas without ulceration (right).

137 We also examined whether ulceration status, as indicated by the original pathology report, 
$0.56,1.00]$ was achieved for melanomas with ulceration and an $\mathrm{AUC}=0.71[95 \% \mathrm{Cl}: 0.50$, 0.92] for melanomas without ulceration (Fig 2A, right). The opposite trend was observed with the NRAS model, where ulcerated melanomas led to a decreased AUC to $0.45[95 \%$ $\mathrm{Cl}$ 0.07-0.83] and non-ulcerated melanomas had an increased $\mathrm{AUC}$ to $0.92[95 \% \mathrm{Cl}$ : ROls that excluded areas of ulceration, indicating that the network is not learning from the presence of an ulceration on the slide itself. Rather, elements in the tumor microenvironment that influence the ulceration status are potentially playing an important role in determining NRAS mutation status.

In order to confirm that tumor thickness and ulceration alone are not predictors of mutational status, we built a multivariate logistic regression model where Breslow depth and ulceration status are the predictive variables for the presence of mutated BRAF or NRAS. The model was trained on the same training dataset used for our deep CNN. This model performed at random for predicting either mutated BRAF and NRAS, with $\mathrm{AUC}=0.53[95 \% \mathrm{Cl}: 0.34,0.72]$ and $\mathrm{AUC}=0.52[95 \% \mathrm{Cl}: 0.30,0.75]$, respectively (Supplemental Figure 3). This demonstrates that the necessary features for predicting 156 mutation status are provided by the histopathological slide. Performance for the mutation network can be visualized with a probability heat map, where the presence of the mutation of interest is shown in red and intensity of color corresponding to the probability of mutation. Fig. 3A demonstrates representative H\&E sections of melanomas with their corresponding probability heat maps for BRAF-mutant 
162 (left), and 2 correctly identified non-BRAF-mutant tumors: e.g. NRAS-mutant (center) and

163 WT/WT melanoma (right), respectively. Similarly, Fig. 3B demonstrates probability heat

164 maps for the NRAS prediction network, with correctly identified NRAS-mutant (left) and

165 non-NRAS-mutant melanoma. Interestingly, in both BRAF-mutant (center) and WTMT

166 melanomas (right), there are regions identified by the network to harbor mutated NRAS,

167 raising the possibility of intratumoral heterogeneity.

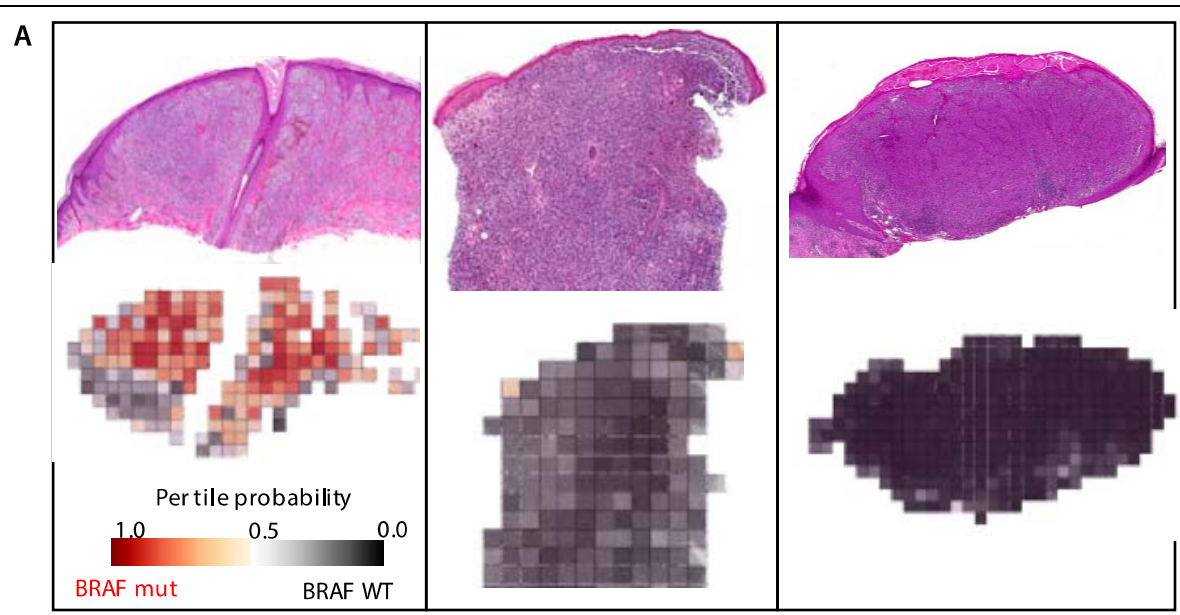

B

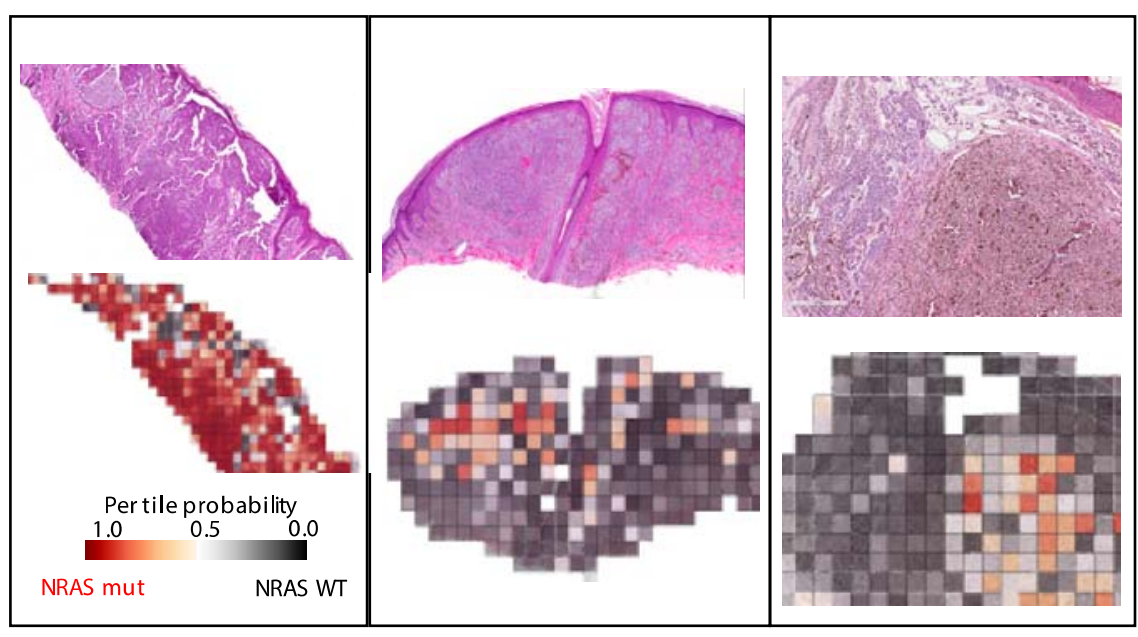

Figure 3. Probability heat maps for mutation prediction A) H\&E slide section and corresponding heat map of a correctly classified BRAF mut melanoma (left), NRAS ${ }^{\text {mut }}$ melanoma (center) and WT/WT melanoma (right) slides by the BRAF mutation prediction network. B) H\&E slide section and corresponding heat map of a correctly classified NRAS ${ }^{\text {mut }}$ melanoma (left), BRAF ${ }^{\text {mut }}$ melanoma (center) and WT/WT melanoma (right) slides by the NRAS mutation prediction network. 
171 Predicting mutated BRAF and NRAS using images from The Cancer Genome Atlas

172 database

173 An image dataset of digitized FFPE H\&E-stained slides of primary melanomas were

174 retrieved from TCGA, a collaborative and publicly available research database comprised

175 of tumor tissue and genomic data from multiple cancer types ${ }^{9}$. This dataset was used as

176 an independent cohort, which comprised of 40 BRAF-mutant cases, 9 NRAS-mutant

177 cases, and 22 WT/WT cases, summing up to 71 cases in total. After quality control, 68

178 images were approved for the independent validation.

180 Breslow depth information was available for 32 out of the 68 slides $^{15}$. Melanomas from

181 the TCGA database were skewed towards very thick tumors with a median of $7.5 \mathrm{~mm}$.

182 There were no tumors less than $1.0 \mathrm{~mm}$ in depth. In comparison, the median depths of

183 tumors in our training, validation and test cohorts were 2.00, 1.45, and 1.90, respectively

184 (Supplemental Figure 4). AUCs of mutation prediction were calculated for melanomas

$185 \leq 1.0 \mathrm{~mm},>1.0-5.0 \mathrm{~mm},>5.0-10 \mathrm{~mm},>10-15 \mathrm{~mm}$, and $>15-20 \mathrm{~mm}$ for NYU and TCGA

186 cohorts (Supplemental Table 2). For BRAF mutation prediction on TCGA images, the

187 network performed better for melanomas $\leq 5 \mathrm{~mm}$. AUC values decreased with thicker

188 tumors. This trend is similar to the effect tumor thickness had on our NYU test cohort (Fig.

189 4A). Tumor thickness did not affect network predictive ability for mutated NRAS on TCGA

190 tumors, as was previously observed in our own cohort (Supplemental Table 2). 
Regarding the effect of ulceration on network performance, TCGA melanomas without ulceration (i.e., T2a, T3a and $\mathrm{T} 4 \mathrm{a}, \mathrm{n}=10$ ) were compared to melanomas with ulceration (i.e., T2b, T3b and T4b, n=56). The ulceration status of TCGA tumors did not have a significant impact on BRAF mutation prediction (Supplemental Table 3), consistent with our observations in the NYU cohort. Importantly, network performance for predicting

197 mutated NRAS was significantly enhanced for non-ulcerated melanomas with an $\mathrm{AUC}=0.89$ [95\% Cl: 0.67-1.0] compared to $\mathrm{AUC}=0.45$ [95\% Cl: 0.24-0.67] for ulcerated melanomas, reproducing the difference that ulceration status has on NRAS mutation prediction in our own cohort (Fig. 4B, Supplemental Table 3).
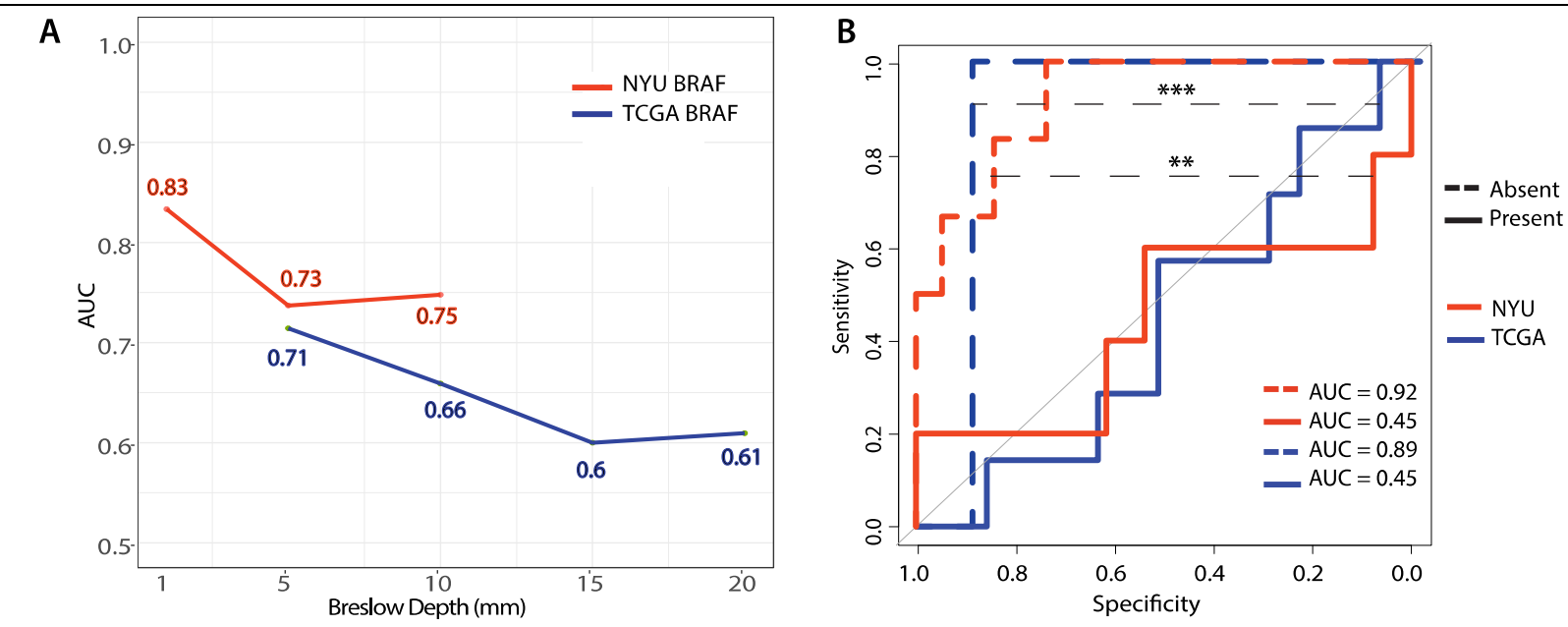

Figure 4. Validation of network performance on an independent cohort from TCGA. A) AUC variation for different Breslow depth values on the independent NYU test set and the TCGA cohort. $B R A F$ mutation prediction is improved for thinner melanomas both on the NYU test set and the TCGA external validation cohort. B) NRAS mutation prediction is improved for melanomas without ulcerations both on the NYU independent cohort and on the TCGA external validation cohort. automate the identification of melanoma by processing tiled images as "in" the ROI or 
and a per tile AUC=0.92 [95\% Cl: 0.922,0.924] (Fig. 5A). H\&E-stained non-annotated whole slides of BRAF-mutant, NRAS-mutant and WTMT melanomas are shown in Fig.

5B-D. along with their corresponding network-generated probability heat maps, where orange indicates tumor, gray indicates non-tumor, and the intensity of the color correlates with the probability gradient. Notably, there is excellent concordance between the pathologist and the network. Network performance was independent of melanoma

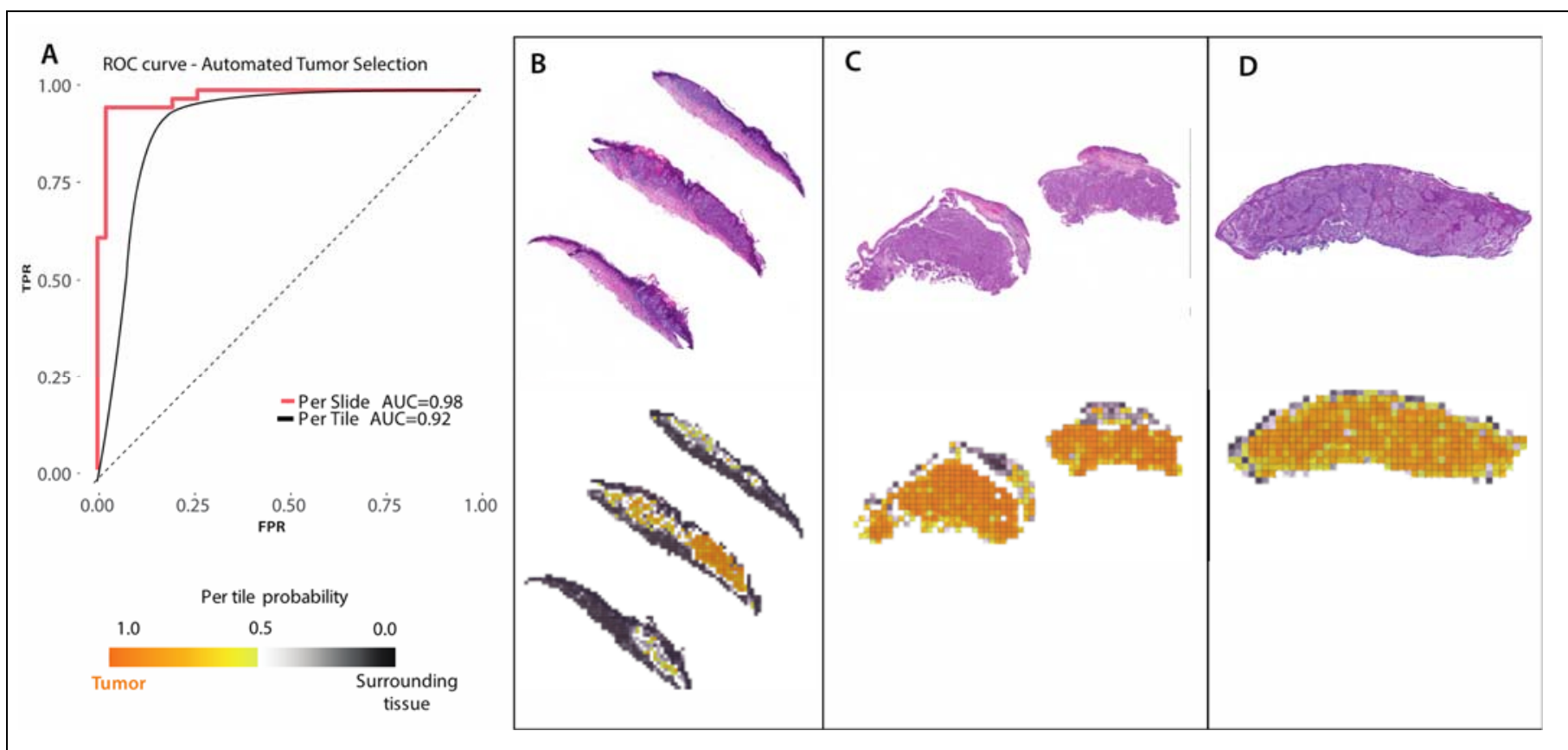

Figure 5. Automating tumor selection for a fully automated sequential workflow A) ROC and AUC for the automated tumor selection network. H\&E slide section and corresponding heat map of tumor annotation on B) a BRAF-mutated slide C) a NRAS-mutated slide and D) a WT/WT slide.

218 We then examined whether the automated tumor selection network could be combined

219 with the mutation prediction networks in a sequential manner. The computational

220 workflow as outlined in Fig. 1. was repeated on all non-annotated images that were tiled 
221 and passed through the automated tumor selection network. Tiles assigned with a probability of belonging to tumor area (probability $>=0.5$ ) were filtered and split in training, validation and independent sets. The Inception v3 architecture was re-trained on tiles selected by the automated network for mutation prediction. Importantly, the 44 slides that comprised the independent set for the algorithm trained on manually annotated images were maintained as the independent set for the algorithm trained on network selected tumors. In this fully automated network, model performance achieved an AUC=0.75 [95\% $\mathrm{Cl}: 0.58,0.89]$ for predicting mutated $B R A F$ and an $\mathrm{AUC}=0.70[95 \% \mathrm{Cl}: 0.47,0.90]$ for predicting mutated NRAS (Supplemental Figure 6). These results are consistent with model performance trained on the manual annotations, indicating that the automated

231 tumor selection network performs as well as the dermatopathologist and does not impact 232 mutation prediction performance. Fig. 6. outlines the complete workflow of mutation 233 prediction for melanoma H\&E histopathology slides using sequential networks for 234 automated tumor selection and mutation prediction.

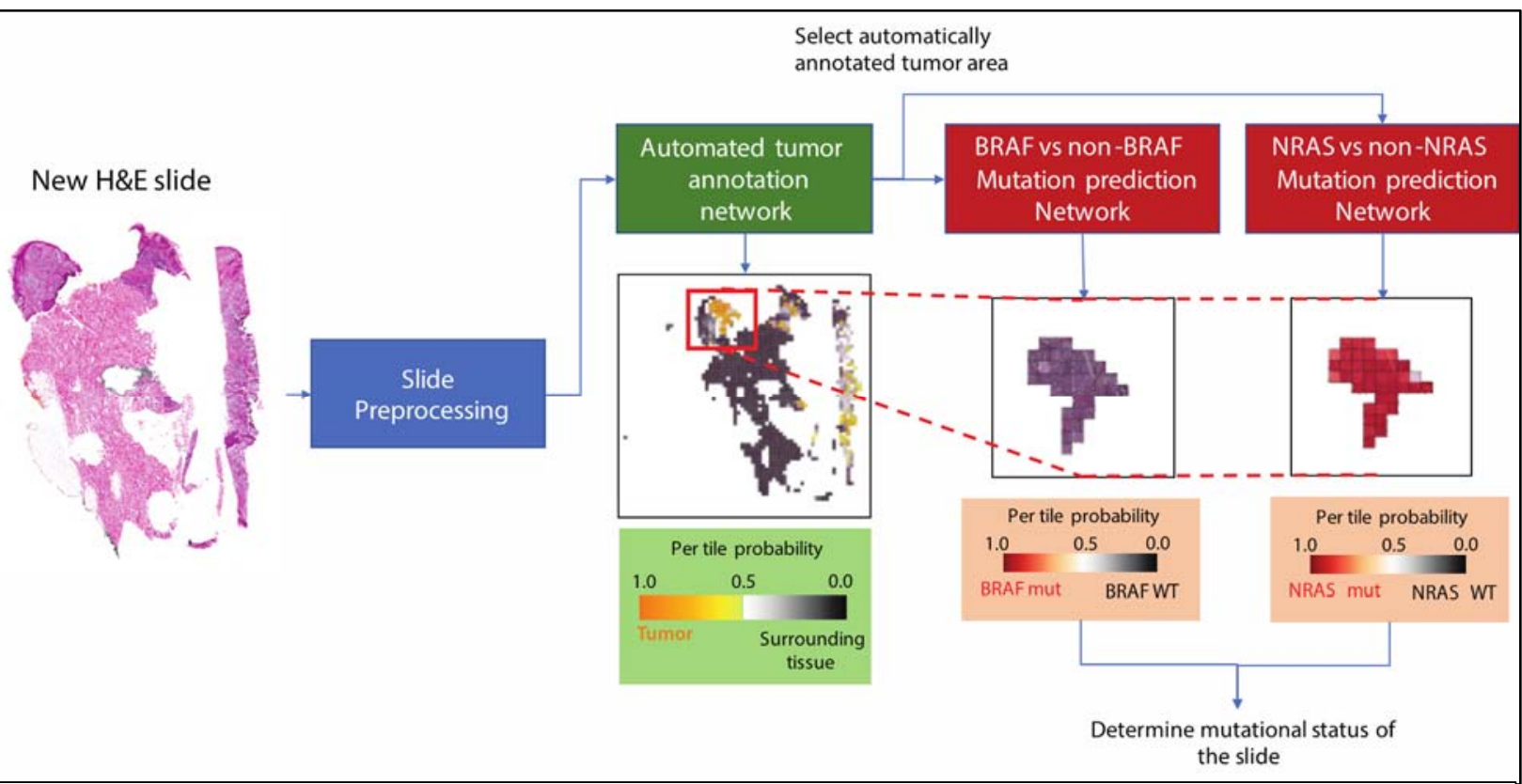

Figure 6. Fully Automated Sequential Workflow. Non-annotated whole slides are processed, tiled, and passed through the automated tumor annotation network which assigns a probability to each tile of belonging in the tumor. Tiles with probability $>=0.5$ are subsequently passed through the mutation prediction network for determining the mutational status of the slide of interest. 
Association of network mutation localization with immunohistochemical analysis

237 To further corroborate network accuracy, we examined whether network-generated

238 probability heat maps are true visual representations of mutation localization. An

239 additional set of $39 B R A F^{V 600 E}$ cases underwent automated algorithmic prediction and

240 immunohistochemical (IHC) analysis with the monoclonal VE1 antibody, a reliable

241 screening tool for detecting the specific V600E mutation ${ }^{16}$. The tumor selection algorithm

242 was applied with a threshold of 0.1 to remove tiles with very low tumor probability. The

243 top 10 cases predicted to be $B R A F^{M u t}$ were subjected to IHC analysis for BRAFV600E

244 staining. Regions of positive IHC staining were manually annotated by a single

245 dermatopathologist blinded to the mutation status of the cases. In Fig. 7A, the annotated

246 mask of positive IHC staining was overlaid on the network-generated probability heat map

247 for our highest confidence prediction. The average probability of tiles falling inside vs.

248 outside the selected mask was calculated (see Methods) and displayed as the

249 corresponding box plot in Fig. 7B. Tiles containing BRAFMut were significantly more likely

250 to fall within the IHC mask compared to outside the mask $(p<3 e-08)$, indicating that the

251 network indeed localizes mutated BRAF. Similar results were obtained for the top 10

252 ranked predicted $B R A F^{V 600 E}$ cases, for the majority of which there was statistically

253 significant concordance between the heat map and IHC (Fig. 7C). Surprisingly, IHC failed

254 to detect mutated BRAF in two of these ten high confidence cases (Fig. 7D). 


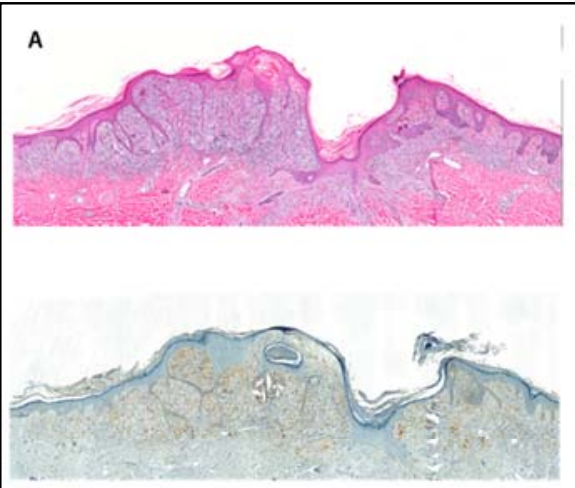

c

C BRAF V600E Per tile Probability Distribution
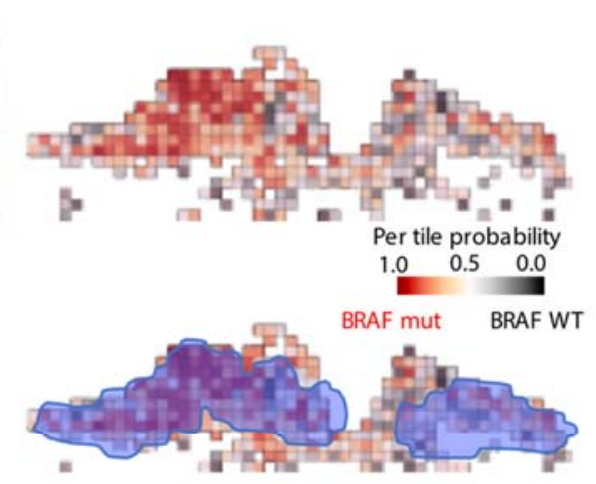

$=$

B

BRAF V600E Per tile

Probability Distribution

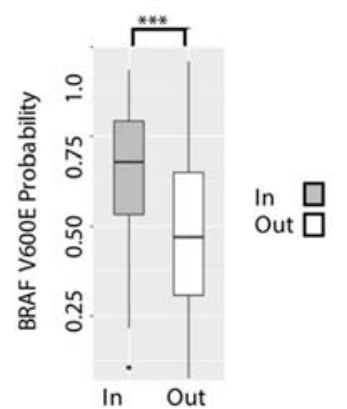

Relative tile location to the mask applied by dermatopathologist
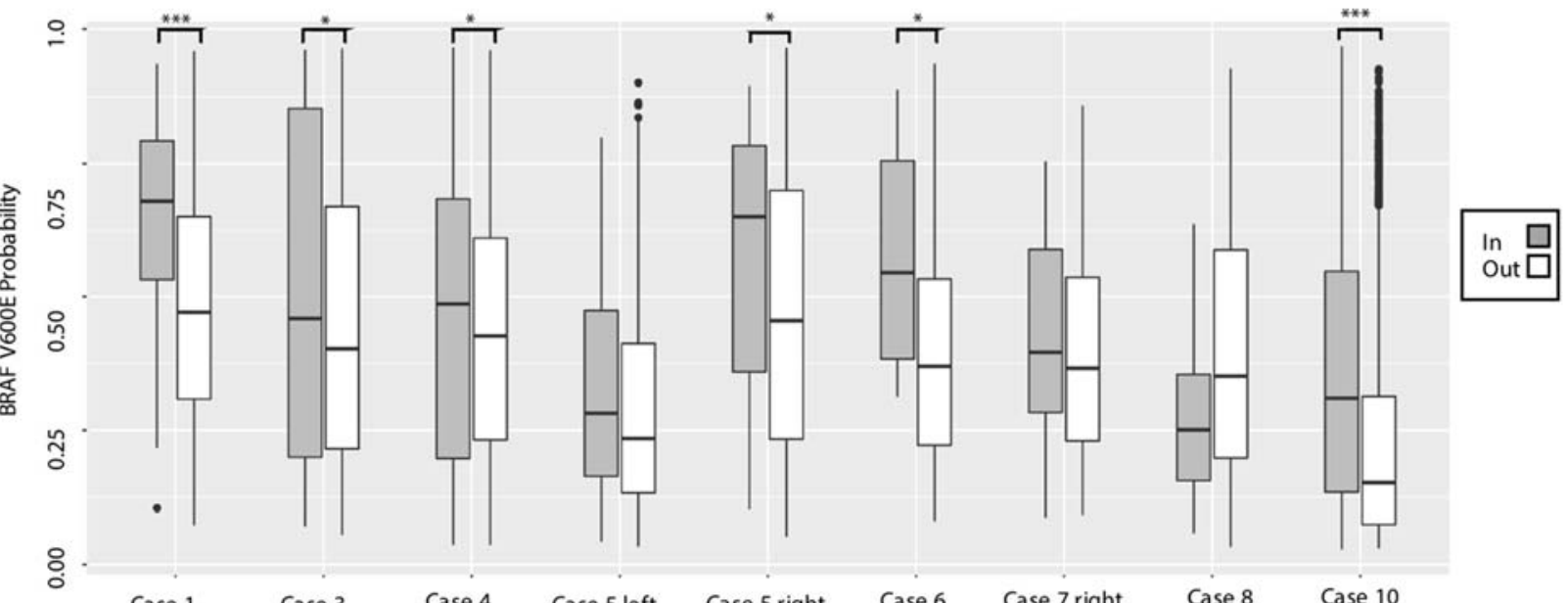

D
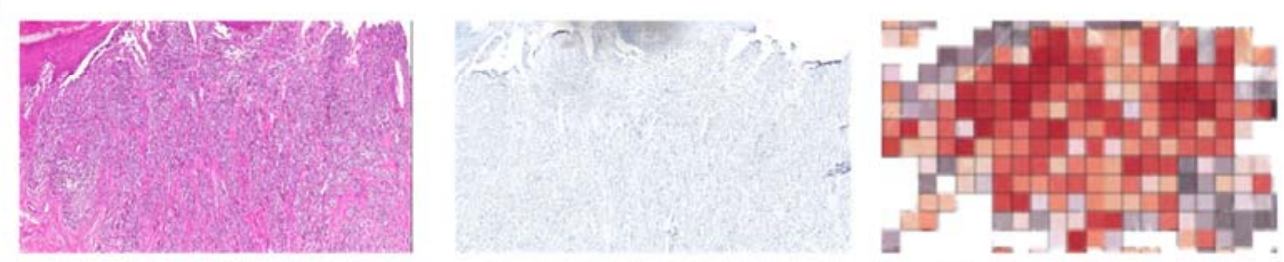

Per tile probability
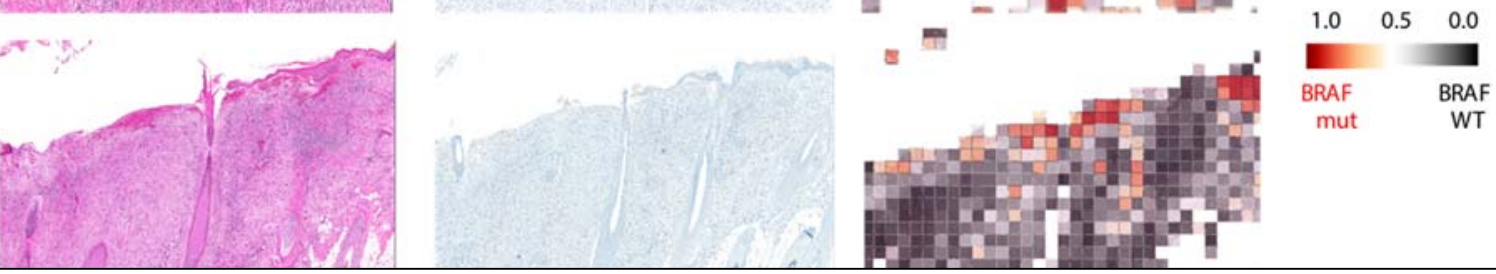

Figure 7. BRAF V600E-predicted tumor areas overlap with immunohistochemical V600E antibody staining. A) Annotated regions of positive IHC staining demonstrating overlap with the network-generated probability heat map. H\&E-stained tissue section (top left), IHC-stained tissue section (bottom left), probability heat map (top right), and overlay (bottom right) are shown. B) Boxplot of the probability distributions for tiles inside and outside the IHC mask. Tiles predicted to harbor $B R A F^{M u t}$ were more likely to fall inside the IHC mask $(p<3 e-09)$. C) Boxplot distributions and IHC-heat map overlays of high-probability $B R A F^{\text {Mut }}$ cases. P-values for statistically significant cases: Case 1 : $p=9 e-08$; Case 3: $p=0.03$; Case 5, right: $p=0.012$; Case 6: $p=0.018$; Case 10: $p=6.6 e-16$. D) Case 2 with representative H\&E-stained section, IHC-stained section, and probability heat map and Case 9 with representative $\mathrm{H} \& \mathrm{E}$-stained section, IHC-stained section, and probability heat map. 


\section{Discussion}

262 In melanoma, deep learning has previously been applied to classify pigmented lesions as benign vs. malignant using clinical ${ }^{17}$ or dermoscopic ${ }^{18}$ images with impressive accuracy. Nevertheless, histopathological examination remains the gold standard for the diagnosis of melanoma. In patients with localized disease, surgical excision is curative. For advanced melanomas, the development of targeted therapies, such as BRAF and MEK inhibitors, and immunotherapies, such as anti-CTLA4 and anti-PD1 antibodies, have substantially increased median overall survival ${ }^{3}$. Selecting the optimal treatment in these patients depends, in part, on determining the mutational status of the BRAF oncogene. While the ideal treatment regimen for NRAS-mutated melanomas is still unclear, combination therapy with MEK inhibition is under investigation ${ }^{3}$. Mutational testing is therefore routinely performed on Stage III and IV melanomas. Here, we use a deep learning approach on whole slide histopathology images to predict for BRAF and NRAS driver mutations in primary melanomas.

Specific morphologic signatures associated with mutated BRAF have been described independently with dermoscopy ${ }^{19}$, reflectance confocal microscopy ${ }^{20}$, and histology ${ }^{14,21}$.

278 Histologic features include greater pagetoid scatter, intraepidermal nesting, epidermal 279 thickening, better circumscription, larger rounder and more pigmented melanocytes, and 280 less solar elastosis. However, attempts to develop binary decision trees to predict for the BRAF mutation using histology alone achieved a predictive accuracy of only $60.3 \%{ }^{21}$. In our study, we corroborate that BRAF mutations lead to specific morphologic changes that can be detected through deep learning and demonstrate that network performance for 
284 predicting mutated $B R A F$ is improved with thinner tumors with an accuracy of $83 \%$. As

285 several of the morphologic features described to be specific for mutated BRAF

286 predominantly affect the epidermis, it may be the case that it is easier for the network to

287 detect these features in thinner tumors. In studies correlating BRAF mutations and tumor

288 thickness, some have found BRAF mutations to be associated with thinner tumors ${ }^{22}$ while

289 others have reported either an inverse 23,24 or no relationship ${ }^{25,26}$ with Breslow depth.

291 Evaluating the effect of ulceration status on our institutional cohort suggests an association between ulceration and mutated BRAF melanomas, as there was a modest improvement in AUC for predicting mutated BRAF in ulcerated melanomas. In a logistic regression model using clinicopathological features, only ulceration and histologic subtype were found to be significant predictors for mutated $B R A F^{26}$. Ulceration may be due to downregulation of genes involved in cell adhesion pathways through copy number losses on chromosomes $6 q$ and 10q. Losses at 10q23-26 have been connected with BRAF mutations ${ }^{27}$, providing support for an association with an ulcerative state.

So far, NRAS-mutated melanomas have non-specific histologic findings, such as greater 301 mitotic index ${ }^{28}$, fewer tumor-infiltrating lymphocytes ${ }^{29}$, and nodular histologic subtypes ${ }^{30}$. 302 Not surprisingly, attempts to predict for NRAS mutation using pre-defined morphologic 303 features performed at random ${ }^{14,21}$ and studies examining NRAS-mutated melanomas and parameters such as tumor thickness and ulceration have yielded conflicting results ${ }^{25}$ 31, 32. Using deep learning, we demonstrate that NRAS mutations can be predicted from histopathology images, indicating that these specific morphologic features have not yet 
307 been fully described. It is possible these features are detectable on the nuclear or chromosomal level, as NRAS mutations more frequently exhibit chromosomal loss of the 11q23.3-11q25 region, whereas BRAF mutations are associated with loss at 10q23-26 and gains at chromosome 7 and $1 q 23-q 25^{27}$. This provides a structural basis that could explain, in part, how our deep learning methods are able to classify these mutations.

312 Intriguingly, in non-ulcerated melanomas, network performance to predict mutated NRAS 313 reached $\mathrm{AUC}=0.92$ in our institutional cohort. Ulceration may represent a distinct

314 biological subtype as it is the second most significant prognostic factor in melanoma 315 survival. In addition to genetic alterations, it has been proposed that the tumor-infiltrating 316 lymphocytes (TILs) are a critical factor in ulcerated melanomas ${ }^{33}$. The importance of TILs 317 for prognosis and response to treatment is an area of active investigation in 318 immunooncology; and deep learning has been used to create spatial maps of TILs and 319 correlating TIL patterns with survival ${ }^{34}$. Thus, there may be contributions from the tumor microenvironment that influence network performance in a more substantial manner for NRAS-mutated melanomas. a reduced performance compared to our institutional cohort. TCGA primary melanoma specimens are enriched for thicker tumors, with a median of $2.7 \mathrm{~mm}$ and a mean of $4.9 \mathrm{~mm}^{15}$. We verified that this cohort contained thicker melanomas, with a median depth of $7.5 \mathrm{~mm}$ at the time of initial diagnosis. Our network model was trained on melanomas with a more equitable distribution among all tumor stages (Supplemental Figure 3). Nevertheless, we were able to corroborate some of the observed trends with subgroup 
analysis. Although there were no TCGA melanomas thinner than $1.0 \mathrm{~mm}$, network performance for predicting mutated $B R A F$ was greatest in melanomas $\leq 5.0 \mathrm{~mm}$, with a

332 continual reduction of performance as tumor thickness increased. Interestingly, 333 extrapolating BRAF network performance on the TCGA dataset in Fig 4A leads to a 334 predicted AUC of approximately 0.80 on melanomas $\leq 1.0 \mathrm{~mm}$, similar to the AUC of 0.83 335 obtained on our institutional cohort. In addition, we again found that the absence of an 336 ulceration is an important factor for predicting mutated NRAS, with an $A U C=0.89$ on 337 TCGA images.

With respect to existing rapid screening tests, it is unclear to what extent immunohistochemistry is being used in clinical practice. Although antibodies to detect $341 B R A F^{V 600 E}$ and NRASQ61R specific mutations have reported high sensitivities and specificities $^{5}$, known limitations of interpretation include: variations in staining, equivocal 343 or ambiguous staining in tumors with high melanin content $(>10 \%)$, samples with $<10 \%$ 344 of tumor content ${ }^{35}$, and false negatives in inappropriately fixed tissue. Furthermore, 345 despite shorter turnaround times ${ }^{6}$, sample preparation and slide cutting still incur additional time and cost. For these reasons, IHC requires optimized and standardized testing protocols ${ }^{36}$ and interpretation of results by an experienced pathologist.

349 In our study, we utilize IHC analysis with the monoclonal VE1 antibody to further 350 substantiate the accuracy of our model by assessing the overlay between positive IHC 351 staining of $B R A F^{V 600 E}$ on tissue sections and network-generated probability heat maps. 352 In 10 high probability $B R A F^{\text {Mut }}$ cases, 6 cases demonstrated excellent concordance 
353 between positive IHC staining and the heat map, 2 cases showed no statistically

354 significant overlap, and 2 cases were misidentified by IHC as negative. One of these false

355 negative cases was noted by the pathologist to contain high amounts of background

356 pigment, highlighting certain advantages deep learning may have over current screening

357 methods.

Because whole slide image analysis will be a crucial feature for clinical adaptability, we fully automated our mutation prediction by first applying a tumor selection model on nonannotated images, achieving an $\mathrm{AUC}=0.98$. The high discriminatory power of our model mutation status (Supplemental Figure 5). Importantly, the performance of the fully automated model was comparable to the manual annotation model across all our analyses.

With the recent FDA approval of the first whole slide imaging system for primary diagnosis in pathology ${ }^{37}$, the digitization of slides seems poised to be integrated into routine clinical practice. In the context of our mutation prediction model, primary melanomas could be rapidly screened on initial H\&E slides. While we did not utilize metastatic melanoma samples in this study over concerns of suboptimal training on a smaller dataset, a number

372 of studies demonstrate mutational testing on the primary tumor is an acceptable 373 alternative $38,39,40$. Our BRAF model could potentially be used in conjunction with IHC 374 screening, where concordant cases do not require confirmatory sequencing. 375 Alternatively, cases that were negative for the BRAFV600E mutation by IHC can be 
analyzed by deep learning in order to identify false negatives or non-V600E BRAF mutants in patients who would benefit from targeted therapy. As others have advocated using multiple detection methods for challenging samples ${ }^{41}$ or to minimize techniquerelated discordancy ${ }^{42}$, additional rapid and cost-effective mutational screening techniques would be highly valuable. Regarding our NRAS model, additional training with an increased sample size at $40 x$ image magnification can improve overall network performance. Intriguingly, our network performs particularly well on non-ulcerated, NRASmutated melanomas, suggesting there may be contributions in the tumor microenvironment that warrant further study.

There is great promise for advanced computational approaches to be integrated into clinical care. Beyond predicting mutations, our study lays the groundwork for more sophisticated deep learning models based on histopathology images, such as predicting for treatment responders vs. non-responders or even survival outcomes, as has been previously demonstrated in lung cancers ${ }^{43}$ and gliomas ${ }^{44}$. We present a fully automated deep CNN model that accurately differentiates melanomas from benign tissue and uses morphologic features to predict the presence of $B R A F$ or NRAS driver mutations. Not only has this approach provided additional insight into how these mutations may affect tumor structural characteristics and its surrounding environment, our models have the potential to complement existing mutation screening assays, with the advantage of significantly reducing costs, and importantly, expediting the decision-making process for treatment. 


\section{Materials and Methods}

400

401

402

403

404

405

406

407

408

409

410

411

412

413

414

415

416

417

418

419

420

421

Dataset of whole-slide images

All patients were enrolled in an IRB-approved clinicopathological database and biorepository in the Interdisciplinary Melanoma Cooperative Group (IMCG) at NYU Langone Health. The IMCG collects prospective clinical, pathological, and follow-up data from melanoma patients who present for diagnosis and/or treatment ${ }^{45} .365$ H\&E-stained FFPE whole-slides from 324 primary melanomas diagnosed between 1994 to 2013 were retrieved and digitized at 20x magnification. A single board-certified dermatopathologist (RHK) reviewed all digitized slides for image quality and excluded images that were blurry, faded, or did not contain any tumor. 293 images from 257 melanomas were subsequently annotated by RHK for tumor-rich regions of interest (ROIs) using Aperio ImageScope software. Driver mutations were previously determined by Sanger sequencing.

\section{Software availability}

We utilized the adapted Tensorflow pipeline (https://github.com/ncoudray/DeepPATH.git) to perform our analysis using the Inception v3 CNN architecture.

\section{Image pre-processing}

Whole-slide images were partitioned at 20x magnification into non-overlapping 299x299 pixel "tiles". This process generated 794,588 total tiles in our dataset, after removing tiles with more than $50 \%$ background (white area of slides). All tiles take the label of the slide

21 they belong to and are sorted in training, validation and independent sets comprising of 
$42270 \%, 15 \%$ and $15 \%$ of the total number of tiles correspondingly. Tiles of images coming

423 from the same patient are all included in the same set. Tiles in the train and validation

424 sets were then converted to TF record format, which is necessary for training of Inception

$425 \mathrm{v} 3$, in groups of 1024 tiles in each TF record file for the training set and 128 tiles for the

426 validation set.

429 The Inception v3 architecture is a Convolutional Neural Network (CNN) that utilizes modules comprised of various convolutions with different kernel sizes and a max pooling layer. The network was trained on $70 \%$ of the tiles from each data set, with $15 \%$ of the tiles used for validation and $15 \%$ used for independent testing. The network was trained for maximum 500,000 iterations on batches of 30 images with a step of 5,000 iterations.

434 The activation function used in the output layer was softmax. The network's performance was monitored based on the precision on the validation set. The best performing model was chosen when the difference between the precision of the current model and the

437 minimum precision of the previous 5 models was less than 0.01 , indicating a plateau in precision. The performance of the best model was then evaluated on the independent set (44 slides) and the AUC was calculated. The network outputs a probability value for every tile for each class of interest. The tile is assigned to the class with the highest probability. A heat map for each slide in the test set is generated. The color intensity is analogous to the probability value of the tile to belong in each class. 


\section{Network performance on the data from The Cancer Genome Atlas}

71 FFPE slides of primary melanomas from the TCGA were downloaded and tiled into non-overlapping tiles of 299x299 pixels. All tiles were sorted for testing and TFRecord files were generated. The slides were passed through the mutation prediction networks and the average probabilities per slide were used for the AUC calculation.

\section{Automated tumor selection}

Whole images were tiled in non-overlapping tiles of 299x299 pixels. The tiles were sorted based on their position compared to the manual tumor selection applied by the dermatopathologist as 'in' and 'out' tumor, and were divided in train, validation and independent sets the same way as for the mutation prediction networks. The same 44 slides were kept as the independent set. The Inception v3 model was trained on these two classes of tiles and the performance of the best performing model on the validation set was measured on the independent set.

\section{Annotated and automated mutation prediction}

For the annotated model, only tiles belonging inside the annotated tumor area were taken into consideration. For the automated model, only tiles belonging inside the tumor area as determined by the tumor annotation network were selected for training. The tiles were sorted in two categories depending on the mutation classification task and based on the true label of the slide they belong to. They were also divided in train, validation and independent set as before. Inception v3 was trained on the tiles and performance was 
467 monitored based on the precision on the validation set. The best performing model was

468 obtained and evaluated on the independent set.

Sequential network

471 To apply the sequential model, non-annotated slides of interest were tiled in non472 overlapping 299x299 pixel tiles. The tiles were first passed through the automated tumor selection network which will output a probability for each tile belonging in the tumor area.

474 Tiles with probability of belonging in the tumor higher or equal than 0.5 were obtained and passed through the BRAF and NRAS mutation prediction networks to assess the mutational profile of the slide.

Statistical analysis

After training and choosing the best performing model on the validation set, model performance was evaluated using the independent dataset, which is comprised of a heldout population of tiles coming from 44 slides. The probabilities for each slide were aggregated by the average of probabilities of the corresponding tiles or by the percentage corresponding Area Under the Curve (AUC) were generated as a measure of accuracy. Heat maps allowed visualization of probability differences and regions of interest.

Multivariate model

488 The multivariate logistic regression model was built using the $g / m$ function in $R$ from the 489 package ROCR. 
Receiver Operating Characteristic Curves

ROC curves were generated using the pROC package in $R$ and the $p$-values were calculated using the roc.test() function. archival melanoma sample sections collected on plus slides (Fisher Scientific, Cat\# 22042-924) and stored at room temperature. Unconjugated, mouse anti-human SerineGR32335840-6) raised against a synthetic peptide within human BRAF (amino acids 550650) containing the glutamic acid substitution, was used for $\mathrm{IHC}^{38,46}$. BRAF antibody was

502 optimized on known positive and negative colon samples and subsequently validated on was performed on a Ventana Medical Systems Discovery Ultra using Ventana's reagents and detection kits unless otherwise noted. In brief, slides were deparaffinized online and antigen retrieved for 24 minutes at $95^{\circ} \mathrm{C}$ using Cell Conditioner 1 (Tris-Borate-EDTA pH8.5). BRAF was diluted 1:50 in Ventana antibody diluent (Ventana Medical Systems, Cat\# 251-018) and incubated for 16 minutes at $36^{\circ} \mathrm{C}$. Endogenous peroxidase activity was post-primary blocked with $3 \%$ hydrogen peroxide for 4 minutes. Primary antibody was detected using Optiview linker followed by multimer-HRP incubated for 8 minutes

511 each, respectively. The complex was visualized with 3,3 diaminobenzidene for 8 minutes 512 and enhanced with copper sulfate for 4 minutes. Slides were counterstained online with 
513 hematoxylin for 8 minutes and blued for 4 minutes. Slides were washed in distilled water,

514 dehydrated and mounted with permanent media. Positive and negative (diluent only)

515 controls were run in parallel with study sections. Blinded analysis of staining was

516 performed by a single dermatopathologist (GJ).

518 Calculation of BRAF V600E-predicted tumor areas overlap with immunohistochemical 519 V600E antibody staining.

520 By looking at the relative positioning between the IHC and H\&E slides, a direction of shift 521 was chosen and the slides were shifted by a small shift of 1 or maximum 2 tiles towards

522 the observed direction to better align the slides for overlap. Next, the probability 523 distributions for the tiles falling into the mask applied by a dermatopathologist to select 524 for the V600E antibody stained area and the probabilities of the ones outside of the mask 525 were generated. The $\mathrm{p}$ value was calculated using a one-sided Wilcoxon rank sum test. 526 The $p$ values were also adjusted for the number of potential conformations for each slide 527 (shift by 1 , shift by 2 and no-shift) by multiplication with a factor of 3 (multiple testing 528 correction). 


\section{Supplementary Materials}

533 Fig. S1. Mutation prediction classifiers with manual annotation.

534 Fig. S2. Multivariate logistic regression model evaluating Breslow depth and ulceration 535 as predictors for mutational status.

536 Fig S3. Distribution of Breslow Depth for NYU and TCGA cohorts.

537 Fig S4. Performance of automated tumor selection network.

538 Fig. S5. Performance of mutation networks after manual vs. automated tumor selection.

539 Table S1. Distribution of patients and slides within training, validation, and test cohorts.

540 Table S2. Prediction AUC on melanomas stratified by thickness.

541 Table S3. Prediction AUC on melanomas stratified by ulceration. 


\section{References:}

1. Ascierto PA, et al. The role of BRAF V600 mutation in melanoma. J Trans/ Med 10, 85 (2012).

2. Sun J, Zager JS, Eroglu Z. Encorafenib/binimetinib for the treatment of BRAF-mutant advanced, unresectable, or metastatic melanoma: design, development, and potential place in therapy. Onco Targets Ther 11, 9081-9089 (2018).

3. Luke JJ, Flaherty KT, Ribas A, Long GV. Targeted agents and immunotherapies: optimizing outcomes in melanoma. Nat Rev Clin Oncol 14, 463-482 (2017).

4. Cheng L, Lopez-Beltran A, Massari F, MacLennan GT, Montironi R. Molecular testing for BRAF mutations to inform melanoma treatment decisions: a move toward precision medicine. Mod Pathol 31, 24-38 (2018).

5. Barel F, Guibourg B, Lambros L, Le Flahec G, Marcorelles P, Uguen A. Evaluation of a Rapid, Fully Automated Platform for Detection of BRAF and NRAS Mutations in Melanoma. Acta Derm Venereol 98, 44-49 (2018).

6. Bisschop C, et al. Rapid BRAF mutation tests in patients with advanced melanoma: comparison of immunohistochemistry, Droplet Digital PCR, and the Idylla Mutation Platform. Melanoma Res 28, 96-104 (2018).

7. Colomba E, et al. Detection of BRAF p.V600E mutations in melanomas: comparison of four methods argues for sequential use of immunohistochemistry and pyrosequencing. $J$ Mol Diagn 15, 94-100 (2013).

8. Khosravi P, Kazemi E, Imielinski M, Elemento O, Hajirasouliha I. Deep Convolutional Neural Networks Enable Discrimination of Heterogeneous Digital Pathology Images. EBioMedicine 27, 317-328 (2018).

9. Wang Z, Jensen MA, Zenklusen JC. A Practical Guide to The Cancer Genome Atlas (TCGA). Methods Mol Biol 1418, 111-141 (2016).

10. Coudray $\mathrm{N}$, et al. Classification and mutation prediction from non-small cell lung cancer histopathology images using deep learning. Nat Med 24, 1559-1567 (2018).

11. Couture HD, et al. Image analysis with deep learning to predict breast cancer grade, ER status, histologic subtype, and intrinsic subtype. NPJ Breast Cancer 4, 30 (2018).

12. Menzies AM, et al. Intrapatient homogeneity of BRAFV600E expression in melanoma. Am J Surg Pathol 38, 377-382 (2014).

13. Cormican D, Kennedy C, Murphy S, Werner R, Power DG, Heffron C. High concordance of BRAF mutational status in matched primary and metastatic melanoma. J Cutan Pathol 46, 117-122 (2019).

14. Viros A, et al. Improving melanoma classification by integrating genetic and morphologic features. PLoS Med 5, e120 (2008). 
622

623

624

625

626

627

628

629

630

631

632

633

634

635

636

637

638

639

640

641

642

643

644

15. Cancer Genome Atlas N. Genomic Classification of Cutaneous Melanoma. Cell 161, 1681-1696 (2015).

16. Capper D, et al. Assessment of BRAF V600E mutation status by immunohistochemistry with a mutation-specific monoclonal antibody. Acta Neuropathol 122, 11-19 (2011).

17. Esteva A, et al. Dermatologist-level classification of skin cancer with deep neural networks. Nature 542, 115-118 (2017).

18. Haenssle HA, et al. Man against machine: diagnostic performance of a deep learning convolutional neural network for dermoscopic melanoma recognition in comparison to 58 dermatologists. Ann Oncol 29, 1836-1842 (2018).

19. Armengot-Carbo M, Nagore E, Garcia-Casado Z, Botella-Estrada R. The association between dermoscopic features and BRAF mutational status in cutaneous melanoma: significance of the blue-white veil. J Am Acad Dermatol, (2018).

20. Colombino M, et al. Dermoscopy and confocal microscopy for metachronous multiple melanomas: morphological, clinical, and molecular correlations. Eur J Dermatol 28, 149156 (2018).

21. Broekaert SM, et al. Genetic and morphologic features for melanoma classification. Pigment Cell Melanoma Res 23, 763-770 (2010).

22. Liu W, et al. Distinct clinical and pathological features are associated with the BRAF(T1799A(V600E)) mutation in primary melanoma. J Invest Dermatol 127, 900-905 (2007).

23. Ponti G, et al. BRAF, NRAS and C-KIT Advanced Melanoma: Clinico-pathological Features, Targeted-Therapy Strategies and Survival. Anticancer Res 37, 7043-7048 (2017).

24. Mitchell B, Leone DA, Feller JK, Yang S, Mahalingam M. BRAF and epithelialmesenchymal transition in primary cutaneous melanoma: a role for Snail and Ecadherin? Hum Pathol 52, 19-27 (2016).

25. Heppt MV, et al. Prognostic significance of BRAF and NRAS mutations in melanoma: a German study from routine care. BMC Cancer 17, 536 (2017).

26. Spathis A, et al. BRAF Mutation Status in Primary, Recurrent, and Metastatic Malignant Melanoma and Its Relation to Histopathological Parameters. Dermatol Pract Concept 9, 54-62 (2019).

27. Lazar $\mathrm{V}$, et al. Marked genetic differences between BRAF and NRAS mutated primary melanomas as revealed by array comparative genomic hybridization. Melanoma Res 22, 202-214 (2012).

28. Devitt B, et al. Clinical outcome and pathological features associated with NRAS mutation in cutaneous melanoma. Pigment Cell Melanoma Res 24, 666-672 (2011). 
672

673

674

675

676

677

678

679

680

681

682

683

684

685

686

687

688

689

690

691

692

693

694

695

29. Thomas NE, et al. Association Between NRAS and BRAF Mutational Status and Melanoma-Specific Survival Among Patients With Higher-Risk Primary Melanoma. JAMA Oncol 1, 359-368 (2015).

30. Lee JH, Choi JW, Kim YS. Frequencies of BRAF and NRAS mutations are different in histological types and sites of origin of cutaneous melanoma: a meta-analysis. $\mathrm{Br} \mathrm{J}$ Dermatol 164, 776-784 (2011).

31. Si L, et al. Prevalence of BRAF V600E mutation in Chinese melanoma patients: large scale analysis of BRAF and NRAS mutations in a 432-case cohort. Eur J Cancer 48, 94100 (2012).

32. Ellerhorst JA, et al. Clinical correlates of NRAS and BRAF mutations in primary human melanoma. Clin Cancer Res 17, 229-235 (2011).

33. de Moll EH, et al. Immune biomarkers are more accurate in prediction of survival in ulcerated than in non-ulcerated primary melanomas. Cancer Immunol Immunother 64, 1193-1203 (2015).

34. Saltz J, et al. Spatial Organization and Molecular Correlation of Tumor-Infiltrating Lymphocytes Using Deep Learning on Pathology Images. Cell Rep 23, 181-193 e187 (2018).

35. Fisher KE, Cohen C, Siddiqui MT, Palma JF, Lipford EH, 3rd, Longshore JW. Accurate detection of BRAF p.V600E mutations in challenging melanoma specimens requires stringent immunohistochemistry scoring criteria or sensitive molecular assays. Hum Pathol 45, 2281-2293 (2014).

36. Long $\mathrm{E}$, et al. Why and how immunohistochemistry should now be used to screen for the BRAFV600E status in metastatic melanoma? The experience of a single institution (LCEP, Nice, France). J Eur Acad Dermatol Venereol 29, 2436-2443 (2015).

37. Evans AJ, et al. US Food and Drug Administration Approval of Whole Slide Imaging for Primary Diagnosis: A Key Milestone Is Reached and New Questions Are Raised. Arch Pathol Lab Med, (2018).

38. Nielsen LB, Dabrosin N, Sloth K, Bonnelykke-Behrndtz ML, Steiniche T, Lade-Keller J. Concordance in BRAF V600E status over time in malignant melanoma and corresponding metastases. Histopathology 72, 814-825 (2018).

39. Manfredi L, et al. Highly Concordant Results Between Immunohistochemistry and Molecular Testing of Mutated V600E BRAF in Primary and Metastatic Melanoma. Acta Derm Venereol 96, 630-634 (2016).

40. Boursault L, et al. Tumor homogeneity between primary and metastatic sites for BRAF status in metastatic melanoma determined by immunohistochemical and molecular testing. PLoS One 8, e70826 (2013).

41. Uguen A, et al. Dual NRASQ61R and BRAFV600E mutation-specific immunohistochemistry completes molecular screening in melanoma samples in a routine practice. Hum Pathol 46, 1582-1591 (2015). 
bioRxiv preprint do: https://doi.org/10.1101/610311; this version posted April 16, 2019. The copyright holder for this preprint (which was not certified by peer review) is the author/funder, who has granted bioRxiv a license to display the preprint in perpetuity. It is made available under aCC-BY-NC-ND 4.0 International license.

696

697

698

699

700

701

702

703

704

705

706

707

708

709

710

711

712

713

714
42. Bruno $\mathrm{W}$, et al. Heterogeneity and frequency of BRAF mutations in primary melanoma: Comparison between molecular methods and immunohistochemistry. Oncotarget 8 , 8069-8082 (2017).

43. $\mathrm{Yu} \mathrm{KH}$, et al. Predicting non-small cell lung cancer prognosis by fully automated microscopic pathology image features. Nat Common 7, 12474 (2016).

44. Mobadersany $\mathrm{P}$, et al. Predicting cancer outcomes from histology and genomics using convolutional networks. Proc Natl Acad Sci U S A 115, E2970-E2979 (2018).

45. Wish LG, et al. Developing a multidisciplinary prospective melanoma biospecimen repository to advance translational research. Am J Transl Res 1, 35-43 (2009).

46. Peris A, Mim MC, Jr., Hoang MP. BAP1 and BRAFV600E expression in benign and malignant melanocytic proliferation. Hum Pathol 46, 239-245 (2015). 
715

716

717

718

719

720

721

722

723

724

725

726

727

728

729

730

731

732

733

734

735

736

737

738
Acknowledgments: We thank Luis Chiriboga from the NYU Experimental Pathology Immunohistochemistry Core Laboratory. The results shown here are in whole or part based upon data generated by the TCGA Research Network: https://www.cancer.gov/tcga. Funding: This research was supported, in part, by the NYU School of Medicine Orbuch-Brand Pilot Grant Program for Cancers of the Skin; by the Laura and Isaac Perlmutter Cancer Center Support Grant; NIH/NCI P30CA016087; and by the National Institutes of Health S10 Grants; NIH/ORIP S10OD01058 and S10OD018338. AT is supported by the American Cancer Society (RSG-15-189-01-RMC). Author contributions: Study concept and design: RHK, SN, IO, AT. Acquisition of data: RHK, SN, ZD, GJ, UM, RLS, RSB. Analysis and interpretation of data: RHK, SN, NC, GJ, JSW, NR, IO, AT; Study supervision: NC, IO, AT. Competing interests: JSW declares the following competing interests:

Stock or Other Ownership: Altor BioScience, Biond, CytomX Therapeutics, Protean Biodiagnostics

Honoraria: Bristol-Myers Squibb, Merck, Genentech, AbbVie, AstraZeneca, Daiichi Sankyo, GlaxoSmithKline, Eisai, Altor BioScience, Amgen, Roche, Ichor Medical Systems, Celldex, CytomX Therapeutics, Nektar, Novartis, Sellas, WindMIL, Takeda, Protean Biodiagnostics

Consulting or Advisory Role: Celldex, Ichor Medical Systems, Biond, Altor BioScience, Bristol-Myers Squibb, Merck, Genentech, Roche, Amgen, AstraZeneca, GlaxoSmithKline, Daiichi Sankyo, AbbVie, Eisai, CytomX Therapeutics, Nektar, Novartis, Sellas, WindMIL, Takeda

Research Funding (to the Institution): Bristol-Myers Squibb, Merck, GlaxoSmithKline, Genentech, Astellas Pharma, Incyte, Roche, Novartis

Travel, Accommodations, Expenses: Bristol-Myers Squibb, GlaxoSmithKline, Daiichi Sankyo, Roche, Celldex, Amgen, Merck, AstraZeneca, Genentech, Novartis, WindMIL, Takeda 
bioRxiv preprint doi: https://doi.org/10.1101/610311; this version posted April 16, 2019. The copyright holder for this preprint (which was not

certified by peer review) is the author/funder, who has granted bioRxiv a license to display the preprint in perpetuity. It is made available under aCC-BY-NC-ND 4.0 International license.

Table 1. Patient characteristics

\begin{tabular}{|c|c|c|c|c|c|c|c|c|c|c|}
\hline \multirow{2}{*}{\multicolumn{2}{|c|}{$\begin{array}{l}\text { Cohorts } \\
\text { Unique Patients (n) }\end{array}$}} & \multicolumn{3}{|c|}{ Training } & \multicolumn{3}{|c|}{ Validation } & \multicolumn{3}{|c|}{ Independent } \\
\hline & & \multicolumn{3}{|c|}{182} & \multicolumn{3}{|c|}{43} & \multicolumn{3}{|c|}{41} \\
\hline \multicolumn{2}{|c|}{ Mutation } & BRAF & NRAS & WT/WT & BRAF & NRAS & WT/WT & BRAF & NRAS & $\begin{array}{l}\text { WT/WT } \\
\end{array}$ \\
\hline & & $\%$ & $\%$ & $\%$ & $\%$ & $\%$ & $\%$ & $\%$ & $\%$ & $\%$ \\
\hline & & $\begin{array}{ll}56 & 30.8\end{array}$ & $\begin{array}{ll}65 & 35.7\end{array}$ & $61 \quad 33.5$ & $14 \quad 32.6$ & $12 \quad 27.9$ & $\begin{array}{ll}17 & 39.5\end{array}$ & $\begin{array}{ll}21 & 51.2\end{array}$ & 22.0 & 26.8 \\
\hline \multirow{3}{*}{$\begin{array}{l}\text { Year of } \\
\text { Diagnosis }\end{array}$} & $<2000$ & 23.6 & 6.2 & 6.2 & $\begin{array}{ll}3 & 21.4\end{array}$ & 0 & 5.9 & $\begin{array}{ll}1 & 4.8\end{array}$ & 0 & 0 \\
\hline & $2001-2010$ & $\begin{array}{ll}50 & 89.3\end{array}$ & $\begin{array}{ll}48 & 73.8\end{array}$ & $\begin{array}{ll}52 & 85.2\end{array}$ & $\begin{array}{ll}8 & 57.1\end{array}$ & $\begin{array}{ll}12 & 100\end{array}$ & $\begin{array}{ll}12 & 70.6\end{array}$ & $\begin{array}{ll}19 & 90.5\end{array}$ & $9 \quad 100$ & $11 \quad 100$ \\
\hline & $>2010$ & 7.1 & $\begin{array}{ll}13 & 20.0\end{array}$ & $\begin{array}{ll}8 & 13.1\end{array}$ & $\begin{array}{ll}3 & 21.4\end{array}$ & 0 & $\begin{array}{ll}4 & 23.5\end{array}$ & 4.8 & 0 & 0 \\
\hline \multicolumn{2}{|c|}{ Age, Avg. +/- Std. } & $\begin{array}{c}59.4+/- \\
16.7\end{array}$ & $\begin{array}{c}61.2+/- \\
17.2\end{array}$ & $\begin{array}{c}65.1+/- \\
14.9\end{array}$ & $\begin{array}{c}53.9+/- \\
19.9\end{array}$ & $\begin{array}{c}70.9+/- \\
16.0\end{array}$ & $\begin{array}{c}68.5+/- \\
14.1\end{array}$ & $\begin{array}{c}59.0+/- \\
14.8\end{array}$ & $\begin{array}{c}59.9+/- \\
14.4\end{array}$ & $\begin{array}{c}65.1+/- \\
12.8\end{array}$ \\
\hline Sex & Female & $\begin{array}{ll}23 & 41.1\end{array}$ & $29 \quad 44.6$ & $\begin{array}{ll}24 & 39.3\end{array}$ & 64.3 & 58.3 & 41.2 & 28.6 & $\begin{array}{ll}6 & 66.7\end{array}$ & 18.2 \\
\hline & Male & $\begin{array}{ll}33 & 58.9\end{array}$ & 55.4 & $\begin{array}{ll}37 & 60.7\end{array}$ & 35.7 & 41.7 & $\begin{array}{ll}10 & 58.8\end{array}$ & $\begin{array}{ll}15 & 71.4\end{array}$ & $\begin{array}{ll}3 & 33.3\end{array}$ & 81.8 \\
\hline Thickness & $<1.01 \mathrm{~mm}$ & 14.3 & 27.7 & $20 \quad 32.8$ & 14.3 & 27.3 & 17.6 & 14.3 & 22.2 & 27.3 \\
\hline & $1.01-2.0 \mathrm{~mm}$ & $\begin{array}{ll}14 & 25.0\end{array}$ & 29.2 & 24.6 & 42.9 & 36.4 & 41.2 & 28.6 & 55.6 & 18.2 \\
\hline & $2.01-4.0 \mathrm{~mm}$ & $\begin{array}{ll}18 & 32.1\end{array}$ & $\begin{array}{ll}20 & 30.8\end{array}$ & $15 \quad 24.6$ & $\begin{array}{ll}5 & 35.7\end{array}$ & 19.1 & 23.5 & $\begin{array}{ll}5 & 23.8\end{array}$ & 111.1 & 36.5 \\
\hline & $>4.0$ & $\begin{array}{ll}16 & 28.6\end{array}$ & 12.3 & $\begin{array}{ll}11 & 18.0\end{array}$ & 7.1 & $\begin{array}{ll}3 & 27.3\end{array}$ & 17.6 & $\begin{array}{ll}7 & 33.3\end{array}$ & $\begin{array}{ll}1 & 11.1\end{array}$ & 18.2 \\
\hline Thickness, $n$ & edian (IQR) & $\begin{array}{c}2.7(1.7- \\
4.6)\end{array}$ & $\begin{array}{c}1.8(1- \\
2.7)\end{array}$ & $\begin{array}{c}1.7(0.9- \\
3.0)\end{array}$ & $\begin{array}{c}1.4(1.2- \\
3.3)\end{array}$ & $\begin{array}{c}1.8(1.0- \\
3.9)\end{array}$ & $\begin{array}{c}1.5(1.1- \\
3.0)\end{array}$ & $\begin{array}{c}2.7(1.6- \\
5.5)\end{array}$ & $\begin{array}{c}1.5(1.0- \\
1.9)\end{array}$ & $\begin{array}{c}2.3(1.1- \\
3.0)\end{array}$ \\
\hline $\begin{array}{l}\text { Histologic } \\
\text { subtype }\end{array}$ & $\begin{array}{l}\text { Superficial } \\
\text { Spreading }\end{array}$ & $\begin{array}{ll}22 & 39.3\end{array}$ & $29 \quad 44.6$ & $\begin{array}{ll}32 & 52.5\end{array}$ & $\begin{array}{ll}8 & 57.1\end{array}$ & $\begin{array}{ll}6 & 50.0\end{array}$ & $\begin{array}{ll}9 & 52.9\end{array}$ & $\begin{array}{ll}9 & 42.9\end{array}$ & $4 \quad 44.4$ & 545.5 \\
\hline & Nodular & $31 \quad 55.4$ & $\begin{array}{ll}34 & 52.3\end{array}$ & $25 \quad 41.0$ & $\begin{array}{ll}6 & 42.9\end{array}$ & $\begin{array}{ll}6 & 50.0\end{array}$ & 47.1 & $12 \quad 57.1$ & $5 \quad 55.6$ & 54.5 \\
\hline & $\begin{array}{l}\text { Lentigo } \\
\text { Maligna }\end{array}$ & 0.0 & 0.0 & 1.6 & 0.0 & 0.0 & 0.0 & 0.0 & 0.0 & 0.0 \\
\hline & Desmoplastic & 5.4 & 1.5 & 4.9 & 0.0 & 0.0 & 0.0 & 0.0 & 0.0 & 0.0 \\
\hline & Unknown & 0.0 & 1.5 & 0.0 & 0.0 & 0.0 & 0.0 & 0.0 & 0.0 & 0.0 \\
\hline Ulceration & Absent & $30 \quad 53.6$ & $\begin{array}{ll}44 & 67.7\end{array}$ & $40 \quad 65.6$ & 64.3 & 58.3 & $\begin{array}{ll}11 & 64.7\end{array}$ & 1152.4 & $\begin{array}{ll}6 & 66.7\end{array}$ & 54.5 \\
\hline & Present & $\begin{array}{ll}25 & 44.6\end{array}$ & $\begin{array}{ll}20 & 30.8\end{array}$ & $\begin{array}{ll}21 & 34.4\end{array}$ & $\begin{array}{ll}5 & 35.7\end{array}$ & $\begin{array}{ll}5 & 41.7\end{array}$ & 35.3 & $\begin{array}{ll}10 & 47.6\end{array}$ & $\begin{array}{ll}3 & 33.3\end{array}$ & 45.5 \\
\hline & Unknown & $\begin{array}{ll}1 & 1.8\end{array}$ & 1.5 & 0.0 & 0.0 & 0.0 & 0.0 & 0.0 & 0.0 & 0.0 \\
\hline Mitotic & Absent & $\begin{array}{ll}8 & 14.3\end{array}$ & 12.3 & $\begin{array}{ll}12 & 19.7\end{array}$ & 14.3 & 8.3 & 11.8 & 19.0 & 0.0 & 9.1 \\
\hline & Few & $\begin{array}{ll}14 & 25.0\end{array}$ & $\begin{array}{ll}23 & 35.4\end{array}$ & $\begin{array}{ll}25 & 41.0\end{array}$ & 7.1 & $\begin{array}{ll}3 & 25.0\end{array}$ & $\begin{array}{ll}5 & 29.4\end{array}$ & $\begin{array}{ll}3 & 14.3\end{array}$ & $\begin{array}{ll}7 & 77.8\end{array}$ & 45.5 \\
\hline & Moderate & $16 \quad 28.6$ & $14 \quad 21.5$ & $\begin{array}{ll}10 & 16.4\end{array}$ & 42.9 & $3 \quad 25.0$ & 29.4 & 23.8 & 111.1 & 18.2 \\
\hline & Many & $\begin{array}{ll}17 & 30.4\end{array}$ & $\begin{array}{ll}20 & 30.8\end{array}$ & $\begin{array}{ll}14 & 23.0\end{array}$ & $\begin{array}{ll}5 & 35.7\end{array}$ & $\begin{array}{ll}5 & 41.7\end{array}$ & $\begin{array}{ll}5 & 29.4\end{array}$ & $\begin{array}{ll}9 & 42.9\end{array}$ & $\begin{array}{ll}1 & 11.1\end{array}$ & 27.3 \\
\hline & Unknown & $\begin{array}{ll}1 & 1.8\end{array}$ & 0.0 & 0.0 & 0.0 & 0.0 & 0.0 & 0.0 & 0.0 & 0.0 \\
\hline AJCC & I & $\begin{array}{ll}15 & 26.8\end{array}$ & $\begin{array}{ll}31 & 47.7\end{array}$ & $\begin{array}{ll}31 & 50.8\end{array}$ & 50.0 & 58.3 & 47.1 & 28.6 & 66.7 & 27.3 \\
\hline Stage & II & $\begin{array}{ll}23 & 41.1\end{array}$ & $21 \quad 32.3$ & $18 \quad 29.5$ & 7.1 & 16.7 & 35.3 & $\begin{array}{ll}9 & 42.9\end{array}$ & $2 \quad 22.2$ & 27.3 \\
\hline & III & $\begin{array}{ll}18 & 32.1 \\
\end{array}$ & $\begin{array}{ll}12 & 18.5 \\
\end{array}$ & $\begin{array}{ll}12 & 19.7 \\
\end{array}$ & 42.9 & 25.0 & 17.6 & 28.6 & 11.1 & 45.5 \\
\hline & IV & 0.0 & 1.5 & 0.0 & 0.0 & 0.0 & 0.0 & 0.0 & 0.0 & 0.0 \\
\hline Anatomic & Axial & $\begin{array}{ll}29 & 51.8 \\
\end{array}$ & 32.3 & 44.3 & 21.4 & 25.0 & 41.2 & 52.4 & 44.4 & 36.4 \\
\hline Site & Extremity & $\begin{array}{ll}17 & 30.4\end{array}$ & 52.3 & 36.1 & $\begin{array}{ll}10 & 71.4\end{array}$ & 75.0 & 52.9 & 33.3 & 55.6 & 18.2 \\
\hline & $\begin{array}{l}\text { Head and } \\
\text { Neck }\end{array}$ & $\begin{array}{ll}10 & 17.9\end{array}$ & $\begin{array}{ll}10 & 15.4\end{array}$ & 19.7 & 7.1 & 0.0 & 5.9 & 14.3 & 0.0 & 45.5 \\
\hline Status & Alive & $\begin{array}{ll}33 & 58.9 \\
\end{array}$ & 58.9 & 58.9 & 58.9 & 58.9 & $12 \quad 58.9$ & 58.9 & 58.9 & 54.5 \\
\hline & $\begin{array}{l}\text { Died of } \\
\text { Melanoma }\end{array}$ & $\begin{array}{ll}20 & 37.0\end{array}$ & $\begin{array}{ll}18 & 27.7\end{array}$ & 25.5 & 45.5 & 30.8 & 5.3 & 47.4 & 112.5 & 71.4 \\
\hline & $\begin{array}{l}\text { Died of Other } \\
\text { Cause }\end{array}$ & 5.1 & 9.7 & 7.6 & 0.0 & 0.0 & 19.0 & 4.5 & 0.0 & 0.0 \\
\hline Recurrence & No & $\begin{array}{ll}31 & 55.4\end{array}$ & 61.5 & 59.0 & 42.9 & 75.0 & $\begin{array}{ll}14 & 82.4\end{array}$ & 42.9 & 77.8 & 18.2 \\
\hline & Yes & 44.6 & $25 \quad 38.5$ & 41.0 & 57.1 & 25.0 & 17.6 & 57.1 & 22.2 & 81.8 \\
\hline
\end{tabular}




\section{Supplemental Material}

A

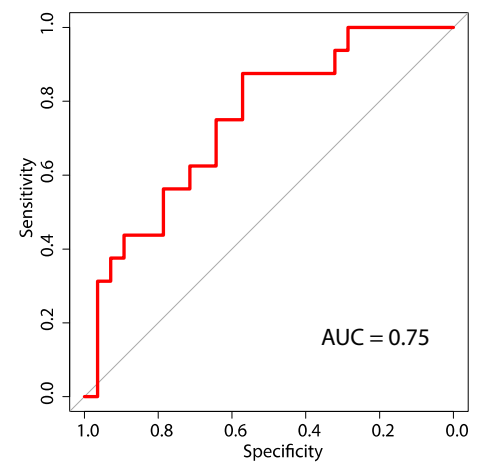

B

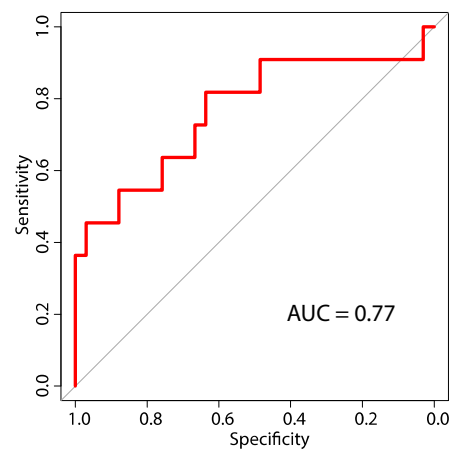

Supplemental Figure 1. Mutation prediction classifiers with manual annotation. A) Receiver Operating Characteristic curve (ROC) and AUC for BRAF vs "non-BRAF" mutation prediction on the entire independent set. B) Receiver Operating Characteristic curve (ROC) and AUC for NRAS vs "nonNRAS" mutation prediction on the entire independent set.

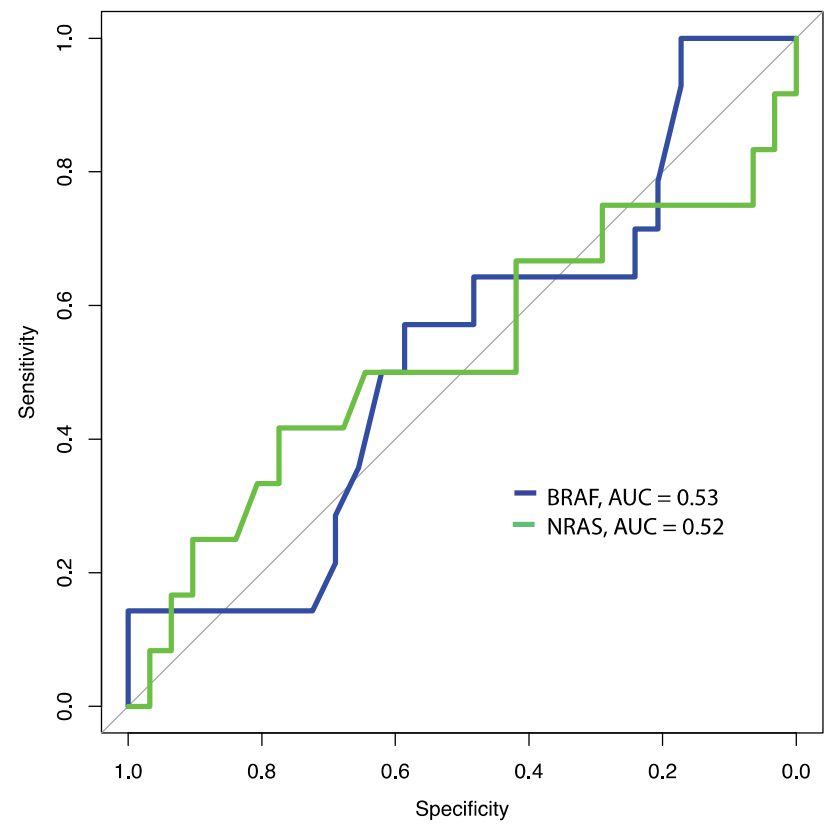

Supplemental Figure 2. ROC curves for the multivariate logistic regression model. The Breslow depth and ulceration variables are not sufficient alone to predict BRAF and NRAS mutations in melanomas, yielding random AUCs. 


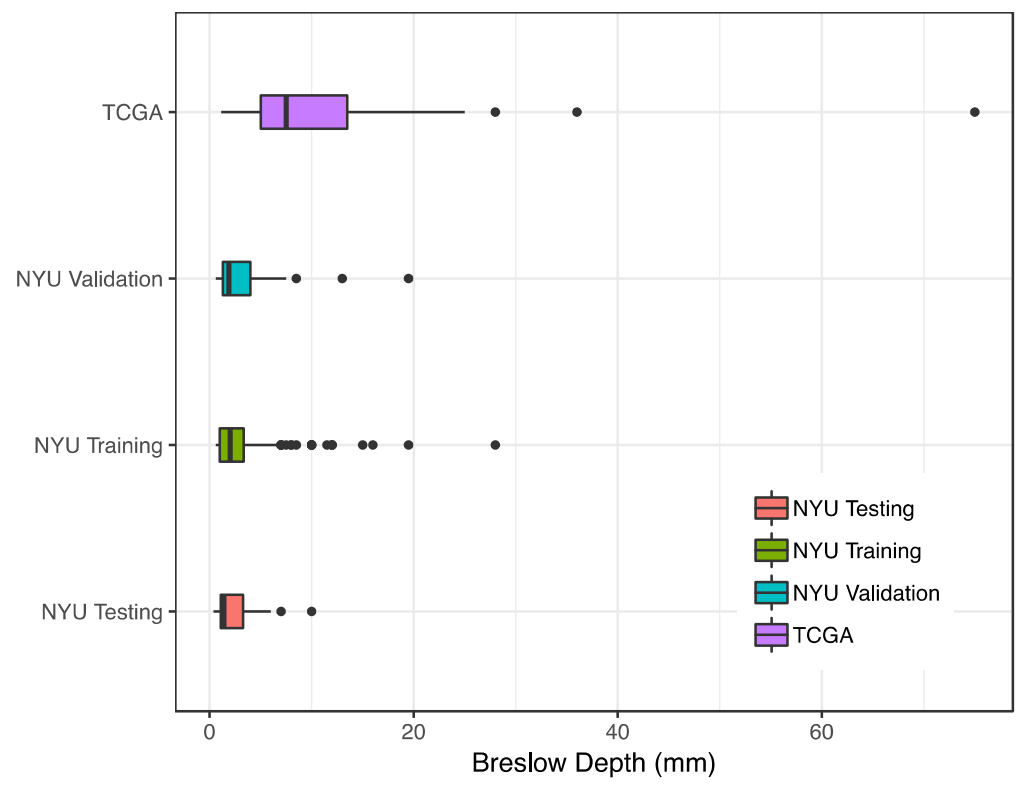

Supplemental figure 3

Breslow depth distribution for our NYU cohorts and the external TCGA validation cohort. It can be observed that the TCGA melanomas are overall much thicker than the ones in our cohort. 
A

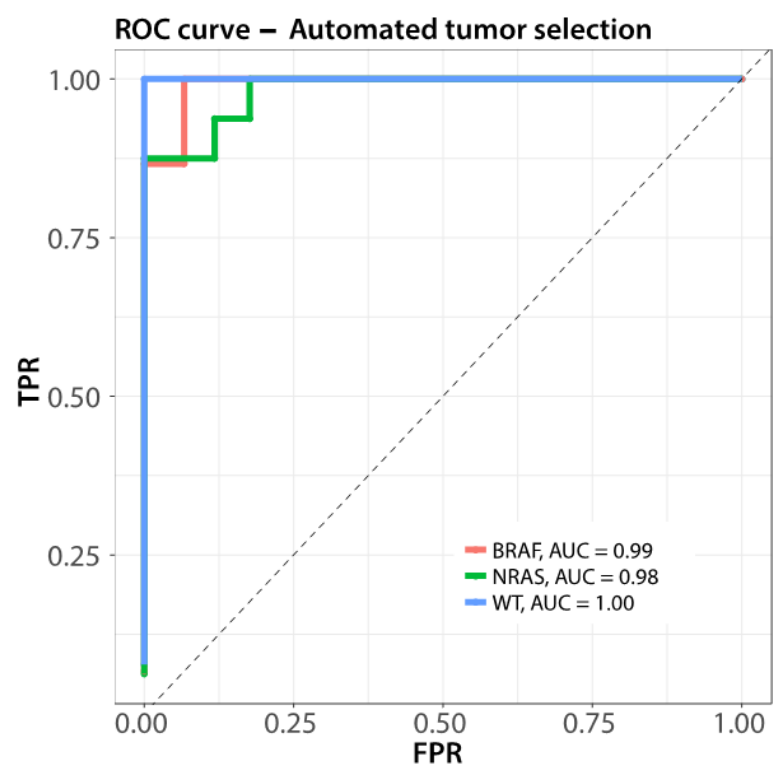

B

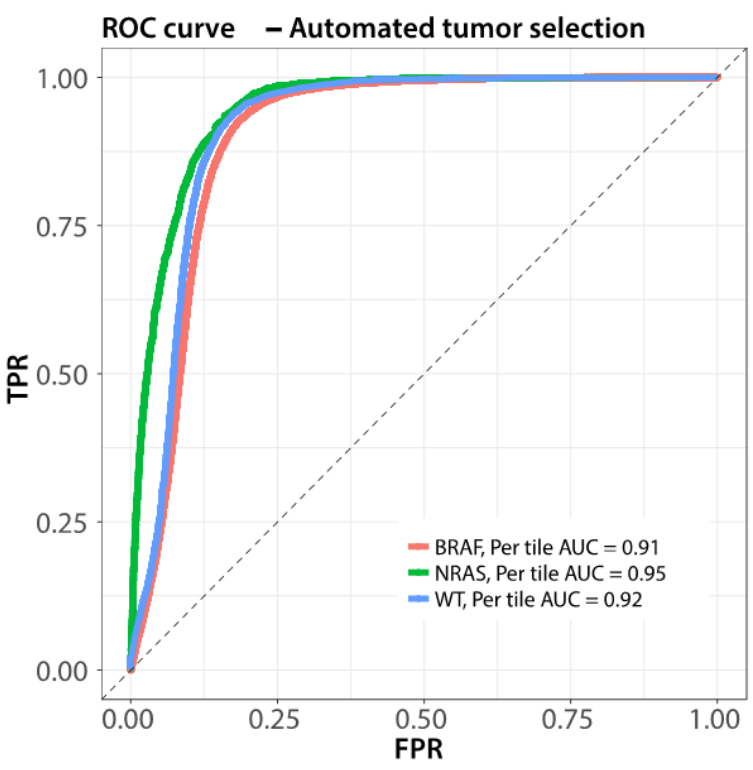

Supplemental Figure 4. Performance of automated tumor selection network.

A) AUC aggregated per slide

B) AUC per tile

A

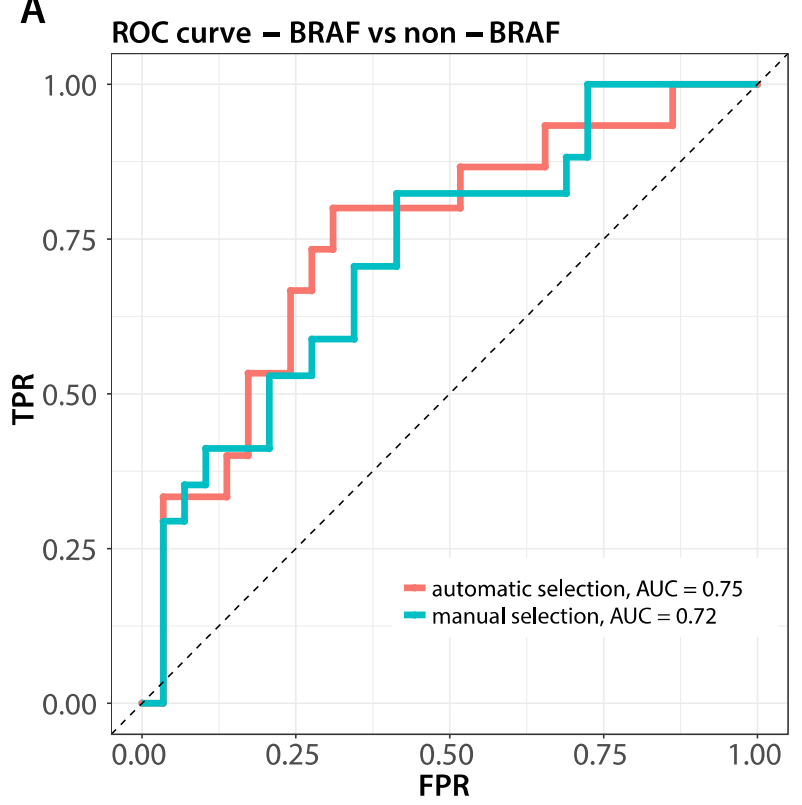

B

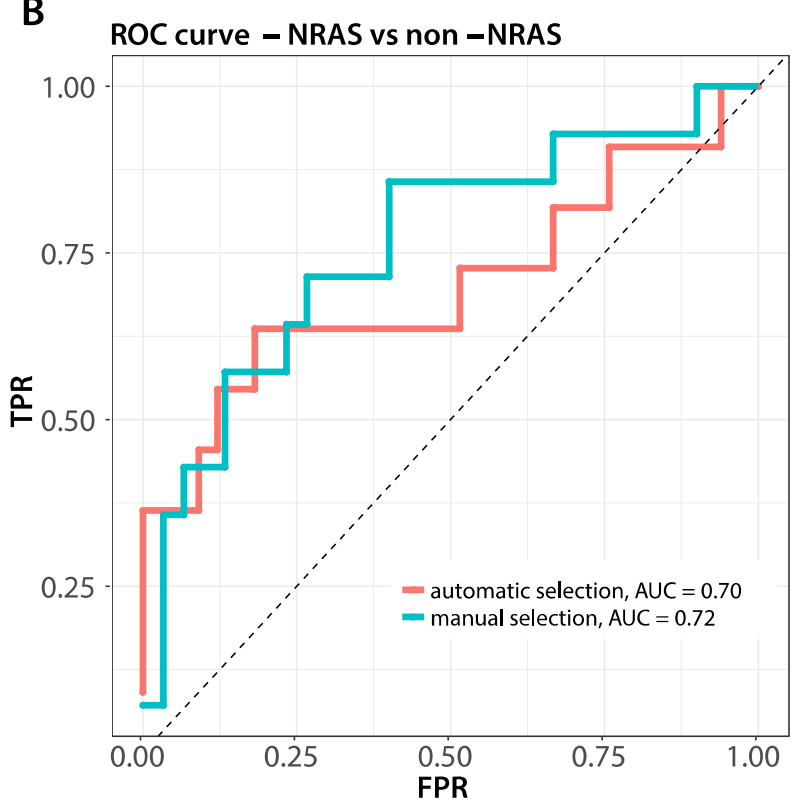

Supplemental Figure 5. Performance of mutation networks after manual vs. automated tumor selection

A) BRAF mutation model

B) NRAS mutation model 


\section{Supplemental Table 1}

The distribution of the number of patients and the corresponding number of slides within each cohort are shown. No patients within the training and validation cohorts overlap with those in the independent cohort.

\begin{tabular}{|l|c|c|c|c|c|c|c|c|}
\hline & \multicolumn{4}{|c|}{ Number of Patients } & \multicolumn{4}{c|}{ Number of slides } \\
\cline { 2 - 9 } & WT & BRAF & NRAS & TOTAL & WT & BRAF & NRAS & TOTAL \\
\hline Train/Validation & 72 & 73 & 75 & 220 & 80 & 87 & 82 & 249 \\
\hline Independent & 15 & 13 & 9 & 37 & 16 & 16 & 12 & 44 \\
\hline TOTAL & 87 & 86 & 84 & 257 & 96 & 103 & 94 & 293 \\
\hline
\end{tabular}

\section{Supplemental Table 2}

$B R A F$ and NRAS prediction AUCs on the independent NYU test set and the TCGA FFPE cohort for different values of Breslow depth.

\begin{tabular}{|c|c|c|c|c|}
\hline & \multicolumn{2}{|c|}{ AUC value for BRAF mutation prediction } & \multicolumn{2}{|c|}{ AUC value for NRAS mutation prediction } \\
\hline Tumor stage & NYU cohort & TCGA cohort & NYU cohort & TCGA cohort \\
\hline$<=1 \mathrm{~mm}$ & $\begin{array}{l}0.83 \\
95 \% \mathrm{CI}[0.45-1]\end{array}$ & - & $\begin{array}{l}0.73 \\
95 \% \mathrm{CI}[0.32-1]\end{array}$ & - \\
\hline$<=5 \mathrm{~mm}$ & $\begin{array}{l}0.74 \\
95 \% \mathrm{CI}[-0.58-0.89]\end{array}$ & $\begin{array}{l}0.71 \\
95 \% \text { CI[0.35-1] }\end{array}$ & $\begin{array}{l}0.84 \\
95 \% \mathrm{CI}[0.70-0.98]\end{array}$ & $\begin{array}{l}0.54 \\
95 \% \mathrm{CI}[0.07-1]\end{array}$ \\
\hline$<=10 \mathrm{~mm}$ & $\begin{array}{l}0.75 \\
95 \% \mathrm{CI}[0.60-0.90]\end{array}$ & $\begin{array}{l}0.66 \\
95 \% \mathrm{CI}[0.41-0.91]\end{array}$ & $\begin{array}{l}0.77 \\
95 \% \text { CI }[0.58-0.96]\end{array}$ & $\begin{array}{l}0.68 \\
95 \% \mathrm{CI}[0.32-1]\end{array}$ \\
\hline$<=15 \mathrm{~mm}$ & - & $\begin{array}{l}0.6 \\
95 \% \mathrm{CI}[0.37-0.83]\end{array}$ & - & $\begin{array}{l}0.66 \\
95 \% \mathrm{CI}[0.40-0.92]\end{array}$ \\
\hline$<=20 \mathrm{~mm}$ & - & $\begin{array}{l}0.61 \\
95 \% \mathrm{CI}[0.39-0.83]\end{array}$ & - & $\begin{array}{l}0.66 \\
95 \% \mathrm{CI}[0.40-0.92]\end{array}$ \\
\hline
\end{tabular}

\section{Supplemental Table 3}

$B R A F$ and NRAS prediction AUCs on the independent NYU test set and the TCGA FFPE cohort for slides with different ulceration status.

\begin{tabular}{|l|l|l|l|l|}
\hline & \multicolumn{2}{|l|}{ AUC value for BRAF mutation prediction } & \multicolumn{2}{l|}{ AUC value for NRAS mutation prediction } \\
\hline Ulceration & NYU cohort & TCGA cohort & NYU cohort & TCGA cohort \\
\hline Present & 0.79 & 0.55 & 0.45 & 0.45 \\
& $95 \% \mathrm{CI}[0.56-1]$ & $95 \% \mathrm{CI}[0.35-0.71]$ & $95 \% \mathrm{CI}[0.07-0.83]$ & $95 \% \mathrm{CI}[0.24-0.67]$ \\
\hline \multirow{2}{*}{ Absent } & 0.71 & 0.6 & 0.92 & 0.89 \\
& $95 \% \mathrm{CI}[0.50-0.92]$ & $95 \% \mathrm{CI}[0.22-0.98]$ & $95 \% \mathrm{CI}[0.81-1]$ & $95 \% \mathrm{CI}[0.67-1]$ \\
\hline
\end{tabular}

